\title{
Tension of the $E_{G}$ statistic and RSD data with Planck/ $\Lambda$ CDM and implications for weakening gravity.
}

\author{
F. Skara* and L. Perivolaropoulos ${ }^{\dagger}$ \\ Department of Physics, University of Ioannina, 45110 Ioannina, Greece
}

(Dated: March 26, 2020)

\begin{abstract}
The $E_{G}$ statistic is a powerful probe for detecting deviations from GR by combining weak lensing (WL), real-space clustering and redshift space distortion (RSD) measurements thus probing both the lensing and the growth effective Newton constants $\left(G_{L}\right.$ and $\left.G_{\text {eff }}\right)$. We construct an up to date compilation of $E_{G}$ statistic data including both redshift and scale dependence $\left(E_{G}(R, z)\right)$. We combine this $E_{G}$ data compilation with an up to date compilation of $f \sigma_{8}$ data from RSD observations to identify the current level of tension between the Planck/ $\Lambda$ CDM standard model based on general relativity and a general model independent redshift evolution parametrization of $G_{L}$ and $G_{e f f}$. Each $f \sigma_{8}$ datapoint considered has been published separately in the context of independent analyses of distinct galaxy samples. However, there are correlations among the datapoints considered due to overlap of the analyzed galaxy samples. Due to these correlations the derived levels of tension of the best fit parameters with Planck/ $\Lambda \mathrm{CDM}$ are somewhat overestimated but this is the price to pay for maximizing the information encoded in the compilation considered. We find that the level of tension increases from about $3.5 \sigma$ for the $f \sigma_{8}$ data compilation alone to about $6 \sigma$ when the $E_{G}$ data are also included in the analysis. The direction of the tension is the same as implied by the $f \sigma_{8}$ RSD growth data alone (lower $\Omega_{m}$ and/or weaker effective Newton constant at low redshifts for both the lensing and the growth effective Newton constants $\left(G_{L}\right.$ and $\left.\left.G_{e f f}\right)\right)$. These results further amplify the hints for weakening modified gravity discussed in other recent analyses [1-4].
\end{abstract}

\section{INTRODUCTION}

The theory of general relativity (GR) and the standard $\Lambda$ cold dark matter $(\Lambda \mathrm{CDM})[5]$ cosmological model have been remarkably successful in explaining a wide array of observations [6] including the observed accelerating expansion of the universe $[7,8]$. Despite of its successes and simplicity, the validity of the cosmological standard model $\Lambda C D M$ is currently under intense investigation. This is motivated by a range of profound theoretical and observational difficulties of the model. The most important theoretical difficulties of the $\Lambda \mathrm{CDM}$ model are the fine tuning [9-11] and coincidence problems $[12,13]$. The first of these problems corresponds to the large discrepancy between observations and quantum field theoretical predictions on the value of the cosmological constant $\Lambda$ while the second is associated with the coincidence between the observed vacuum energy density $\Omega_{\Lambda}$ and the matter density $\Omega_{m}$ which in the present epoch are of the same order of magnitude despite of their very different evolution during the cosmic history.

A well known observational difficulty corresponds to the tension between the cosmic microwave background (CMB) measured value of the Hubble parameter $H_{0}$ $[14,15]$ in the context of the $\Lambda$ CDM model and the local measurements from supernovae $[16,17]$ and lensing time delay indicators [18], with local measurements suggesting a higher value. Another observational puzzle for $\Lambda \mathrm{CDM}$ involves persisting indications from observational probes

\footnotetext{
* fskara@cc.uoi.gr

† leandros@uoi.gr
}

measuring the growth of matter perturbations that the observed growth is weaker than the growth predicted by the standard Planck/ $\Lambda$ CDM parameter values [15]. Modified gravity (MG) models constitute a prime theoretical candidate to explain this tension.

The combination of cosmological observational probes is a powerful tool for the identification of signatures of MG [19-25]. Such observational probes may be divided in two classes: geometric and dynamical (or structure formation) probes [26-29]. Geometric observations measure cosmological distances using standard candles (e.g. Type Ia supernovae) and standard rulers (e.g. the horizon at the time of recombination probed through Baryon Acoustic Oscillations) and thus probe directly the cosmic metric, independent of the underlying theory of gravity. Dynamical observations probe the growth rate of cosmological perturbations and thus the gravitational laws and the consistency of GR with data provided the background geometry is known.

Dynamical probes include cluster counts (CC) [29-32], weak lensing (WL) [25, 33-39] and redshift-space distortions (RSD) [1, 2, 40-42]. These probes are consistent with each other pointing either to a lower value of the matter density parameter $\Omega_{0 m}$ in the context of GR or to weaker gravitational growth power than the growth indicated by GR in the context of a Planck18/ $\Lambda \mathrm{CDM}$ background geometry at about $2-3 \sigma$ level [1, 2, 41]. Such weak growth may be quantified by the parameter $\sigma_{8}$ which is the matter density rms fluctuations within spheres of radius $8 h^{-1} M p c$ and is determined by the amplitude of the primordial fluctuations power spectrum and by the growth rate of cosmological fluctuation.

Various possible mechanisms have been proposed to 
slow down growth at low redshifts and thus reduce the above tension (see e.g. [4]). Such mechanisms may be divided in two categories: non-gravitational and gravitational. The former includes the effects of interacting dark energy models [43-46], dynamical dark energy models $[47,48]$, running vacuum models $[49,50]$ and the effects of massive neutrinos [51]. The latter includes the effects of MG theories with a reduced (compared to GR) evolving effective Newton's constant $G_{\text {eff }}$ at low redshifts [1, 2].

The effects of MG [52-61] models are indistinguishable from GR at the geometric cosmological background level $[26,62,63]$. Signatures of MG can only be obtained by investigating the dynamics of cosmological perturbations $[64,65]$ using specific statistics obtained through dynamical probe observables such as the two-point correlation of and power spectrum of the galaxy distribution, the RSD and WL. A useful bias free statistic is the $f \sigma_{8}$ product of the rate of growth of matter density perturbations $f$ times $\sigma_{8}$ discussed in more detail in what follows. An alternative observable statistic is the $E_{G}$ which was constructed to be independent of both the clustering bias factor $b$ and the parameter $\sigma_{8}$ on linear scales. This statistic was proposed in 2007 [66] and thereafter has been used several times to test MG theories [67, 68]. The expectation value of $E_{G}$ is equal to the ratio of the Laplacian of the sum of the Bardeen potentials [69] $\Psi$ (the Newtonian potential) and $\Phi$ (the spatial curvature potential $) \nabla^{2}(\Psi+\Phi)$ over the peculiar velocity divergence $\theta \equiv \nabla \cdot \frac{\vec{v}}{H(z)}$ (where $\vec{v}$ is the peculiar velocity and $H(z)$ is the Hubble parameter in terms of the redshift $z$ ).

The $E_{G}$ statistic has been proposed as a model independent test of any MG theory [70] and is constructed from three different probes of large scale structure (LSS): the galaxy-galaxy lensing (GGL), the galaxy clustering and the galaxy velocity field which leads to galaxy redshift distortions. Alternatively, $E_{G}$ may be constructed from galaxy-CMB lensing [71] instead of galaxy-galaxy lensing as a more robust tracer of the lensing field at higher redshifts [72, 73].

The first probe, the GGL (a special type of WL), is the slight distortion of shapes of source galaxies in the background of a lens galaxy, which arises from the gravitational deflection of light due to the gravitational potential of the lens galaxy along the line of sight (see for example [74-77]). This WL probe is sensitive to $\nabla^{2}(\Psi+\Phi)$, since relativistic particles collect equal contributions from the two Bardeen potentials which appear in the scalar perturbed Friedmann-Lemaitre-Robertson-Walker (FLRW) metric in the Newtonian gauge [78-80]

$$
d s^{2}=-(1+2 \Psi) d t^{2}+a^{2}(1-2 \Phi) d \vec{x}^{2}
$$

where $a$ is the scale factor that is related to the redshift $z$ through $a=\frac{1}{1+z}$.

The second probe, the galaxy clustering arises from the gravitational attraction of matter and is sensitive only to the potential $\Psi$. Similarly, the third probe, the galaxy velocity field, is quantified by measuring redshift space distortions (RSD) [81-84] (an illusory anisotropy that distorts the distribution of galaxies in redshift space generated by their peculiar motions falling towards overdense regions). This important probe of LSS is sensitive to the rate of growth of matter density perturbations $f$ which depends on the theory of gravity and provides measurements of $f \sigma_{8}$ that depends on the potential $\Psi$.

In most MG theories the potentials $\Phi$ and $\Psi$ obey generalized Poisson equations like the GR Newtonian potential where the MG effects are encoded in generalized space-time dependent effective Newton constants. These generalized Newton constants for the potential $\Psi$ and for the lensing combination $\Psi+\Phi$ are usually described by two parameters: the effective Newton's constant parameter $\mu$ and the light deflection parameter $\Sigma$. In the modified Poisson equations [85] the $\mu$ and $\Sigma$ are connected with the potentials $\Psi$ and $\Psi+\Phi$ respectively. In GR the value of $\mu$ and $\Sigma$ coincides with unity while in a MG model $\mu$ and $\Sigma$ can be in general functions of both time and scale $[19,86]$. Using $f \sigma_{8}$ and $E_{G}$ datasets constraints can be imposed on the parameters $\mu$ and $\Sigma$ [23, 87-92]). Such analyses have revealed various levels of tension of the best fit forms of $\mu$ and $\Sigma$ with the GR prediction of unity showing hints that these parameters may be less than unity implying weaker growth of perturbations than that predicted in GR. The goal of the present analysis is to extend these studies and use an updated data compilation for both the $f \sigma_{8}$ and $E_{G}$ statistics to identify the current level of tension with GR implied by these data compilations.

In particular, we address the following questions:

- What are efficient phenomenological redshift dependent parametrizations of the generalized normalized Newton constants $\mu(z)$ and $\Sigma(z)$ that are consistent with solar system and nucleosynthesis constraints that indicate that GR is restored at high $z$ and at the present time in the solar system?

- What are the constraints imposed by the $E_{G}$ and $f \sigma_{8}$ updated data compilations on the parameters of the above parametrizations and do these constraints amplify the hints for weakening gravity at low $z$ implied by the $f \sigma_{8}$ data alone as indicated by previous studies?

The plan of this paper is the following: In the next Section II we present a brief review of the theoretical expression for $E_{G}$. We also present phenomenologically motivated parametrizations for $\mu$ and $\Sigma$ and describe how we use them in order to probe possible deviations from GR on cosmological scales. In Section III we use compilations of $f \sigma_{8}$ and $E_{G}$ data along with the theoretical expressions for $f \sigma_{8}$ and $E_{G}$ which involve $\mu$ and $\Sigma$ to derive constraints on these parameters and to identify the tension level between the Planck/ $\Lambda \mathrm{CDM}$ parameter values favoured by Planck 2018 [15] shown in Table I and the corresponding parameter values favored by the two datasets. Finally in Section IV we conclude, summarize and discuss the implications and possible 


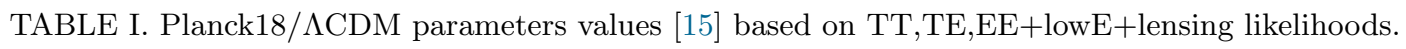

\begin{tabular}{cc}
\hline \hline Parameter & Planck18/ $\mathrm{CDM}$ \\
& \\
\hline$\Omega_{b} h^{2}$ & $0.02237 \pm 0.00015$ \\
$\Omega_{c} h^{2}$ & $0.1200 \pm 0.0012$ \\
$n_{S}$ & $0.9649 \pm 0.0042$ \\
$H_{0}\left[k m s^{-1} M p c^{-1}\right]$ & $67.36 \pm 0.54$ \\
$\Omega_{0 m}$ & $0.3153 \pm 0.0073$ \\
$w$ & -1 \\
$\sigma_{8}$ & $0.8111 \pm 0.0060$ \\
\hline \hline
\end{tabular}

extensions of our analysis.

\section{THEORETICAL BACKGROUND}

\section{II.1. $E_{G}$ statistic}

The $E_{G}$ statistic $[66,70]$ is designed as a probe of the ratio of the Bardeen potentials of the perturbed FRW metric (1.1) in such a way as to be independent of the effects of galaxy bias at linear order. It is defined as the ratio of the cross correlation power spectrum $P_{g \nabla^{2}(\Phi+\Psi)}$ between lensing maps (cosmic shear or CMB) and galaxy positions, over the the cross-correlation power spectrum $P_{g \theta}$ between galaxies and velocity divergence field $\theta$

$$
E_{G} \equiv \frac{P_{g \nabla^{2}(\Phi+\Psi)}}{P_{g \theta}}
$$

In Fourier space the $E_{G}$ statistic may also be expressed as $[66]$

$$
E_{G}(l, \Delta l)=\frac{C_{\kappa g}(l, \Delta l)}{3 H_{0}^{2} a^{-1} \sum_{\alpha} q_{\alpha}(l, \Delta l) P_{v g}^{\alpha}}
$$

where $H_{0}$ is the Hubble parameter today, $l$ is the magnitude of two-dimensional wavenumber of the on-sky Fourier space, $C_{\kappa g}(l, \Delta l)$ is the galaxy-galaxy lensing cross correlation power spectrum in bins of $\Delta l, P_{v g}^{\alpha}$ is the galaxy-velocity cross correlations power spectrum between $k_{\alpha}$ and $k_{\alpha+1}$ (where $k$ three-dimensional wavenumber of the on-sky Fourier transform with $k_{1}<k_{2}<\ldots<$ $\left.k_{\alpha}<\ldots\right)$ and $q_{\alpha}(l, \Delta l)$ is the weighting function defined accordingly.

The corresponding expectation value of $E_{G}$, averaged over $l$ is the the ratio of the Laplacian of the gravitational scalar potentials $\Psi$ and $\Phi$ which appear in the scalar perturbed Friedmann-Lemaitre-Robertson-Walker (FLRW) metric Eq. (1.1) over the peculiar velocity divergence [67]

$$
\left\langle E_{G}\right\rangle=\left[\frac{\nabla^{2}(\Psi+\Phi)}{3 H_{0}^{2} a^{-1} \theta}\right]_{k=l / \bar{\chi}, \bar{z}}
$$

where $\bar{\chi}$ is the comoving mean distance corresponding to the mean redshift $\bar{z}$.
In $\Lambda$ CDM cosmology and assuming that the velocity field is generated under linear perturbation theory, the peculiar velocity divergence is connected to the growth rate $f$ as $\theta=f \delta[93]$ where $\delta \equiv \frac{\delta \rho}{\rho}$ is the matter overdensity field, $\rho$ is the matter density of the background, $f(a) \equiv \frac{d \ln D(a)}{d \ln a}$ is the linear growth rate of structure and $D(a) \equiv \frac{\delta(a)}{\delta(a=1)}$ the growth factor.

In the case of GR and in the absence of any anisotropic stress the Bardeen potentials are equal $(\Psi=\Phi)$ and the gravitational field equations reduce to Poisson equations of the form

$$
\nabla^{2} \Phi=\nabla^{2} \Psi=4 \pi G a^{2} \rho \delta=\frac{3}{2} H_{0}^{2} \Omega_{0 m} a^{-1} \delta
$$

where $\Omega_{0 m}=\Omega_{m}(z=0)$ is the matter density parameter today and the second equality is straightforwardly derived assuming non-relativistic matter species and using the equations $H_{0}^{2}=\frac{8 \pi G \rho_{c, 0}}{3}, \rho=\rho_{0} a^{-3}$ and $\Omega_{0 m}=\frac{\rho_{0}}{\rho_{c, 0}}$ (with $\rho_{0}$ the matter density today and $\rho_{c, 0}$ the critical density today).

Therefore within GR Eq.(2.4), the Eq.(2.3) reduce to

$$
E_{G}=\frac{\Omega_{0 m}}{f(z)}
$$

where $f$ is well approximated as $f(z) \simeq \Omega_{m}^{\gamma}(z)$ with the growth index $\gamma$ in a narrow range near 0.55 , for a wide variety of dark-energy models in GR [94-102]. Note that $E_{G}$ in GR is scale independent (Eq.(2.5)). This is not necessarily the case in the context of MG theories where the growth rate $f$ may be strongly scale dependent even on subhorizon scales.

\section{II.2. The effective Newton's constant parameter $\mu$ and the light deflection parameter $\Sigma$}

The gravitational slip parameter $\eta$ describes the possible inequality $[103,104]$ of the two Bardeen potentials that may occur in MG theories. It is defined as

$$
\eta(a, k)=\frac{\Phi(a, k)}{\Psi(a, k)}
$$


Clearly an observation of $\eta \neq 1$ would indicate physics beyond GR. In this case the gravitational field equations at linear level take the form of Poisson equations that generalize Eqs. (2.4). At linear level, in MG models, using the perturbed metric (1.1) and the gravitational field equations the following phenomenological equations emerge [42, 86, 105-110] for the scalar perturbation potentials

$$
\begin{gathered}
k^{2}(\Psi+\Phi)=-8 \pi G_{N} \Sigma(a, k) a^{2} \rho \Delta \\
k^{2} \Psi=-4 \pi G_{N} \mu(a, k) a^{2} \rho \Delta
\end{gathered}
$$

where $\rho$ is the matter density of the background, $\Delta$ the comoving matter density contrast defined as $\Delta \equiv \delta+$ $3 H a(1+w) v / k$ which is gauge-invariant [106], $w=p / \rho$ is the equation-of-state parameter and $v^{i}=-\nabla^{i} u$ is the irrotational component of the velocity field. Also $\mu$ and $\Sigma$ are the generalized growth and lensing effective Newton constants. They are in general functions of time and scale encoding the possible modifications of General Relativity defined as ${ }^{1}$

$$
\begin{aligned}
\mu(a, k) & \equiv \frac{G_{e f f}(a, k)}{G_{N}} \\
\Sigma(a, k) & \equiv \frac{G_{L}(a, k)}{G_{N}}
\end{aligned}
$$

with $G_{N}$ is the Newton's constant as measured by local experiments, $G_{e f f}$ is the effective Newton's constant which is related to the growth of matter perturbation and $G_{L}$ is related to the lensing of light (the propagation of relativistic particles, such as photons when they traverse equal regions of space and time along null geodesics experiencing gravitational lensing collecting equal contributions from two gravitational potentials $\Psi$ and $\Phi)$. Using the gravitational slip Eq.(2.6) and the ratios of the
Poisson equations (2.7), (2.8) defined above the two LSS functions $\mu$ and $\Sigma$ are related via

$$
\Sigma(a, k)=\frac{1}{2} \mu(a, k)[1+\eta(a, k)]
$$

In GR which predicts a constant homogeneous $G_{\text {eff }}=$ $G_{N}$, we obtain $\mu=1, \eta=1$ and $\Sigma=1$.

Notice that Eqs. (2.7) and (2.8) indicate that a possible observation of reduced gravitational growth of the Bardeen potentials may be interpreted either as reduced strength of gravitational interaction (reduced $\mu$ and/or $\Sigma$ ) or due to reduced matter density $\rho$ (or $\left.\Omega_{0 m}\right)$. In the context of a fixed value of matter density determined by geometric probes of the cosmological background, the reduced gravitational growth could be either interpreted as a tension within the $\Lambda$ CDM parameter value for the matter density or as a hint for weakening gravity. Indeed, such hints of weaker than expected gravitational growth of the Bardeen potentials has been observed at low redshifts by a wide range of dynamical probes including RSD observations [1, 2, 41, 42], WL [25, 34, 36-39] and CC data [29-32]. In most cases this weak growth has been interpreted as a tension for the parameters $\sigma_{8}$ and $\Omega_{0 m}$ which are found by dynamical probes to be smaller than the values indicated by geometric probes in the context of $\Lambda \mathrm{CDM}$.

The observables $f \sigma_{8}(a, k)$ and $E_{G}(a, k)$ can probe directly the gravitational strength functions $\mu(a, k)$ and $\Sigma(a, k)$. In particular $f \sigma_{8}$ is easily expressed in terms of the amplitude $\sigma_{8}$ and the matter overdensity $\delta$ using the matter overdensity evolution equation (see e.g. [80])

$$
\ddot{\delta}+2 H \dot{\delta}-4 \pi G_{N} \mu(a, k) \rho \delta \simeq 0
$$

where the dot denotes differentiation with respect to cosmic time $t$. In terms of redshift Eq. (2.10) takes the form $[1,80]$

$$
\delta^{\prime \prime}(z)+\left(\frac{\left(H(z)^{2}\right)^{\prime}}{2 H(z)^{2}}-\frac{1}{1+z}\right) \delta^{\prime}(z)-\frac{3}{2} \frac{(1+z) \Omega_{0 m} \mu(z, k)}{H(z)^{2} / H_{0}^{2}} \delta(z)=0
$$

where primes denote differentiation with respect to the redshift. While in terms of the scale factor we have [52, 101, 111]

$$
\delta^{\prime \prime}(a)+\left(\frac{3}{a}+\frac{H^{\prime}(a)}{H(a)}\right) \delta^{\prime}(a)-\frac{3}{2} \frac{\Omega_{0 m} \mu(a, k)}{a^{5} H(a)^{2} / H_{0}^{2}} \delta(a)=0
$$

here primes denote differentiation with respect to the scale factor. In Eqs. (2.11), (2.12) possible deviations from GR are expressed by allowing for a scale and

\footnotetext{
1 Note that, in the literature $\mu$ and $\Sigma$ are also referred to as $G_{M}$ and $G_{L}$ (e.g. in Refs. [1, 109]) or as $G_{\text {matter }}$ and $G_{\text {light }}$ (e.g. in Refs. [42, 107]).
}

redshift-dependent $\mu=\mu(z, k)$. In the present section and in section III.1 we ignore scale dependence due to the lack of good quality scale dependent $f \sigma_{8}$ and $E_{G}$ data. However, in section III.2 we discuss the scale dependence of $E_{G}$ data.

For a given parametrization of $\mu(a)$ and initial conditions deep in the matter era where GR is assumed to be valid leading to $\delta \sim a$ equations (2.11), (2.12) may be 
easily solved numerically leading to a predicted form of $\delta(a)$ for a given $\Omega_{0 m}$ and background expansion $H(z)$. In the context of the present analysis we assume a $\Lambda$ CDM backgroung $H(z)$

$$
H^{2}(z)=H_{0}^{2}\left[\Omega_{0 m}(1+z)^{3}+\left(1-\Omega_{0 m}\right)\right]
$$

Once the evolution of $\delta$ is known, the observable product $f \sigma_{8}(a) \equiv f(a) \cdot \sigma(a)$ can be obtained using the definitions

$$
\begin{gathered}
f(a) \equiv \frac{d \ln \delta(a)}{d \ln a} \\
\sigma(a) \equiv \sigma_{8} \frac{\delta(a)}{\delta(a=1)}
\end{gathered}
$$

where $\sigma(a)$ is the redshift dependent rms fluctuations of the linear density field within spheres of radius $R=$ $8 h^{-1} M p c$ and $\sigma_{8}$ is its value today. Thus, we have

$$
f \sigma_{8}\left(a, \sigma_{8}, \Omega_{0 m}, \mu\right)=\frac{\sigma_{8}}{\delta(a=1)} a \delta^{\prime}\left(a, \Omega_{0 m}, \mu\right)
$$

This theoretical prediction may now be used to compare with the observed $f \sigma_{8}$ data and obtain fits for the parameters $\Omega_{0 m}, \sigma_{8}$ and $\mu(z)$ (assuming a specific parametrization of $\mu(z))$.

The lensing gravity parameter $\Sigma(z)$ can be fit in the context of specific parametrizations using its connection with the $E_{G}(a)$ observable as [112-114]

$$
E_{G}\left(a, \Omega_{0 m}, \mu, \Sigma\right)=\frac{\Omega_{0 m} \Sigma(a)}{f\left(a, \Omega_{0 m}, \mu\right)}
$$

This equation assumes that the redshift of the lens galaxies can be approximated by a single value while $E_{G}$ corresponds to average value along the line of sight [114]. In the context of Eq. (2.17) and assuming a specific parametrization for $\mu$ and $\Sigma$, the theoretical prediction for $E_{G}$ may be used to compare with the observed $E_{G}$ datapoints and lead to constraints on $\Omega_{0 m}, \mu, \Sigma$. These constraints may be considered either separately from those of the $f \sigma_{8}$ data or jointly by combining the $E_{G}$ and $f \sigma_{8}$ datasets. The allowed range of these parameters may then be compared with the standard Planck $/ \Lambda \mathrm{CDM}$ parameter values $\mu=1, \Sigma=1, \Omega_{0 m}=0.315 \pm 0.0073, \sigma_{8}=$ $0.811 \pm 0.006$ to identify the likelihood of Planck/ $\Lambda \mathrm{CDM}$ in the context of the dynamical probe data $E_{G}$ and $f \sigma_{8}$ . This plan is implemented in what follows in the context of specific parametrizations describing the possible evolution of $\mu$ and $\Sigma$.

On scales much smaller than the Hubble scale for most modified gravity models the scale dependence of $\mu$ and $\Sigma$ is weak. For example in scalar-tensor (ST) model (for $k \gg a H) \mu$ is independent of the scale [115]. Thus, we start by considering scale independent parametrizations for $\mu$ and $\Sigma$ which reduce to the GR value at early times and at the present time as indicated by solar system (ignoring possible screeing effects) and Big Bang Nucleosynthesis constraints $\left(\mu=1\right.$ and $\mu^{\prime}=0$ for $a=1$ and $\mu=1$ for $a \ll 1$ ) [116-118]. Such parametrizations are of the form $[1,2,119]$

$$
\begin{gathered}
\mu=1+g_{a}(1-a)^{n}-g_{a}(1-a)^{2 n}=1+g_{a}\left(\frac{z}{1+z}\right)^{n}-g_{a}\left(\frac{z}{1+z}\right)^{2 n} \\
\Sigma=1+g_{b}(1-a)^{m}-g_{b}(1-a)^{2 m}=1+g_{b}\left(\frac{z}{1+z}\right)^{m}-g_{b}\left(\frac{z}{1+z}\right)^{2 m}
\end{gathered}
$$

where $g_{a}$ and $g_{b}$ are parameters to be fit and $n$ and $m$ are integer parameters with $n \geq 2$ and $m \geq 2$ which we set equal to 2 in the present analysis.

\section{OBSERVATIONAL CONSTRAINTS}

\section{III.1. Scale Independent Analysis}

The $f \sigma_{8}(z)$ and $E_{G}(z)$ updated data compilations used in our analysis are shown in Tables VI and VII of the Appendix $\mathrm{B}$ along with the references where each datapoint was originally published. The datapoints are also shown in Figs. 1 and 2 along with curves corresponding to the Planck/ $\Lambda \mathrm{CDM}$ prediction and the best fit parameter val- ues. As it can be seen the datapoints from the various surveys are consistent with each other at any given redshift and at $1 \sigma$ level. Clearly, in both cases the data appear to favor lower values of $f \sigma_{8}$ and $E_{G}$ than the values corresponding to the Planck/ $\Lambda \mathrm{CDM}$ parameters. This trend combined with the indications for a Planck/ $\Lambda \mathrm{CDM}$ background from geometric probes may be interpreted as a need for a new degree of freedom which in our approach is coming from MG. In addition, we see that there is no tension between different $f \sigma_{8}$ datapoints. Instead, there is a combined trend of the datapoints to be in tension with the Planck $/ \Lambda \mathrm{CDM}$ prediction. This tension disappears when we keep the same $\Lambda$ CDM background but allow for a MG evolution of the effective Newton's constant. In fact, this trend may be shown to be translated 


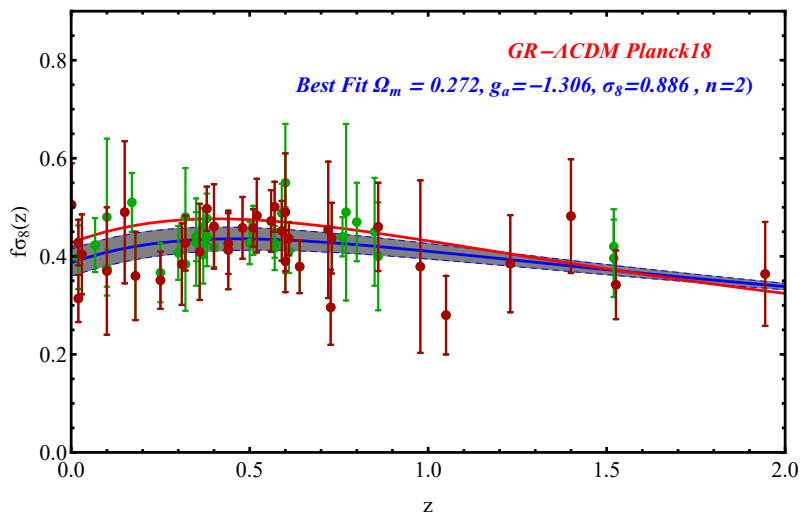

FIG. 1. The $f \sigma_{8}(z)$ data compilation from Table VI used in the present analysis. The subset of the data with less correlation is indicated with dark red. The red curve shows the Planck18/ $\Lambda$ CDM prediction (parameter values $\Omega_{0 m}=0.315$, $\left.g_{a}=0, \sigma_{8}=0.811\right)$, the blue curve shows the best fit of the $f \sigma_{8}(z)$ in the context of parametrizations Eq.(2.18) with a $\Lambda$ CDM background (parameter values $\Omega_{0 m}=0.272$, $\left.g_{a}=-1.306, \sigma_{8}=0.886\right)$ and the shaded regions correspond to $1 \sigma$ confidence level around the best fit (see also Table II).

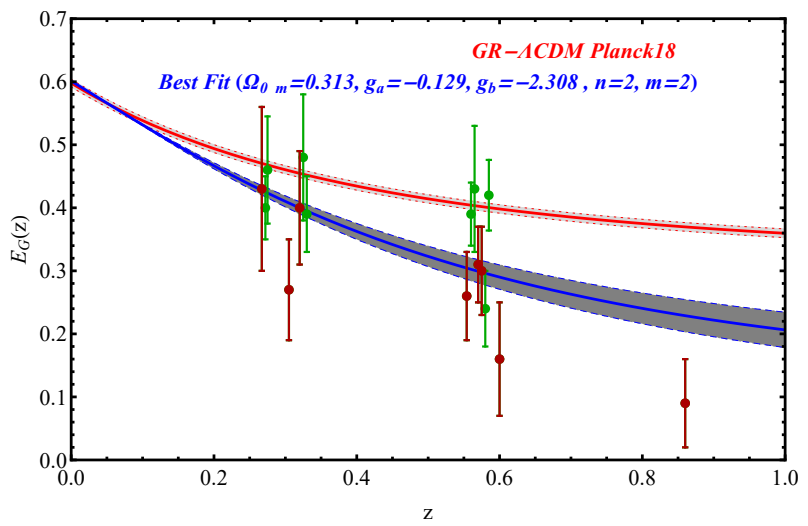

FIG. 2. The $E_{G}(z)$ data compilation from Table VII (scales $3<R<150 h^{-1} \mathrm{Mpc}$ ) used in the present analysis. The subset of the data with less correlation is indicated with dark red. The red curve shows the theoretical prediction based on the Planck18/ $\Lambda$ CDM parameter values $\left(\Omega_{0 m}=0.315\right.$, $\sigma_{8}=0.811, \mu=1, \Sigma=1$ ), the blue curve shows the best fit theoretical prediction based on the parametrizations (2.18) and (2.19) with parameter values $\left(\Omega_{0 m}=0.313, g_{a}=-0.129\right.$, $\left.g_{b}=-2.308\right)$. Notice that the best fit is significantly below the Planck/ $\Lambda \mathrm{CDM}$ theoretical prediction and implies weaker gravity $(\mu<1$ and $\Sigma<1$ ) at the $4.6 \sigma$ level (see also Table II).

into a trend for lower values for the gravitational parameters $\mu$ and $\Sigma$ and is quantified through a detailed maximum likelihood analysis.

Each $f \sigma_{8}(z)$ and $E_{G}(z)$ datapoint of the compilations of Tables VI and VII has been published separately in the context of independent analyses of distinct galaxy samples and lensing data. However, the correlations among the datapoints considered due to overlap of the analyzed galaxy samples may lead to an amplification of the existing trends indicated by the data and an amplification of the existing tension of the best fit parameters with Planck/ $\Lambda \mathrm{CDM}$. Despite of this fact we have chosen to keep the relatively large number of distinct published datapoints in order to maximize the information encoded in the compilations considered keeping in mind that this may lead to an artificial amplification of the trends that already exist in the data.

An additional motivation for keeping the full set of published datapoints is that it is not always clear which one of the correlated points is more suitable to keep. Ignoring one of the correlated points arbitrarily or simply based on time of publication criteria could lead to loss of useful information or selection bias.

Keeping the full set of points does not significantly change the results and the level of tension between the growth data best fit parameter values corresponding to MG and Planck/ $\Lambda$ CDM best fit in the context of GR. In order to demonstrate the validity of the above reasons we have repeated our analysis for a subset of the $f \sigma_{8}$ and $E_{G}$ data where we have removed most earlier data that were subject to correlations with more recent data as indicated with bold font in the index of the Tables VI and VII and as shown in Figs. 1 and 2 with dark red. The result was a data compilation of about half the $f \sigma 8$ and $E_{G}$ datapoints with significantly less correlation. The results of the statistical analysis of this dataset are presented in Appendix A and indicate a minor reduction of the overall tension.

For the construction of the likelihood contours of the model parameters in the context of the $f \sigma_{8}$ and $E_{G}$ datasets we construct $\chi_{f \sigma_{8}}^{2}$ and $\chi_{E_{G}}^{2}$ For the construction of $\chi_{f \sigma_{8}}^{2}$ we use the vector [2]

$$
V_{f \sigma_{8}}^{i}\left(z_{i}, p\right) \equiv f \sigma_{8, i}^{o b s}-\frac{f \sigma_{8}^{t h}\left(z_{i}, p\right)}{q\left(z_{i}, \Omega_{0 m}, \Omega_{0 m}^{f i d}\right)}
$$

where $f \sigma_{8, i}^{o b s}$ is the the value of the $i$ th datapoint, with $i=1, \ldots, N_{f \sigma_{8}}$ (where $N_{f \sigma_{8}}=66$ corresponds to the total number of datapoints of Table VI) and $f \sigma_{8}^{t h}\left(z_{i}, p\right)$ is the theoretical prediction, both at redshift $z_{i}$. The parameter vector $p$ corresponds to the parameters $\sigma_{8}, \Omega_{0 m}, g_{a}$ of Eq. (2.16) with the parametrization (2.18). The fiducial Alcock-Paczynsk correction factor $q[1,2,41]$ is defined as

$$
q\left(z_{i}, \Omega_{0 m}, \Omega_{0 m}^{f i d}\right)=\frac{H\left(z_{i}\right) d_{A}\left(z_{i}\right)}{H^{f i d}\left(z_{i}\right) d_{A}^{f i d}\left(z_{i}\right)}
$$

where $H(z), d_{A}(z)$ correspond to the Hubble parameter and the angular diameter distance of the true cosmology and the superscript fid indicates the fiducial cosmology used in each survey to convert angles and redshift to distances for evaluating the correlation function. As shown in Table II, the effects of this correction factor are less than about $10 \%$ in the derived best fit parameter values. 



FIG. 3. The three $1 \sigma-7 \sigma$ confidence contours in $2 \mathrm{D}$ projected parameter spaces of the parameter space $\left(\Omega_{0 m}, \sigma_{8}, g_{a}\right)$ in the context of parametrization Eq.(2.18) with $n=2$ including the fiducial correction factor Eq. (3.2). The RSD data $f \sigma_{8}(z)$ from Table VI of the Appendix B was used. The third parameter in each contour was fixed to the best fit value. The red and green dots describe the Planck18/ $\Lambda$ CDM best fit and the best-fit values from data.
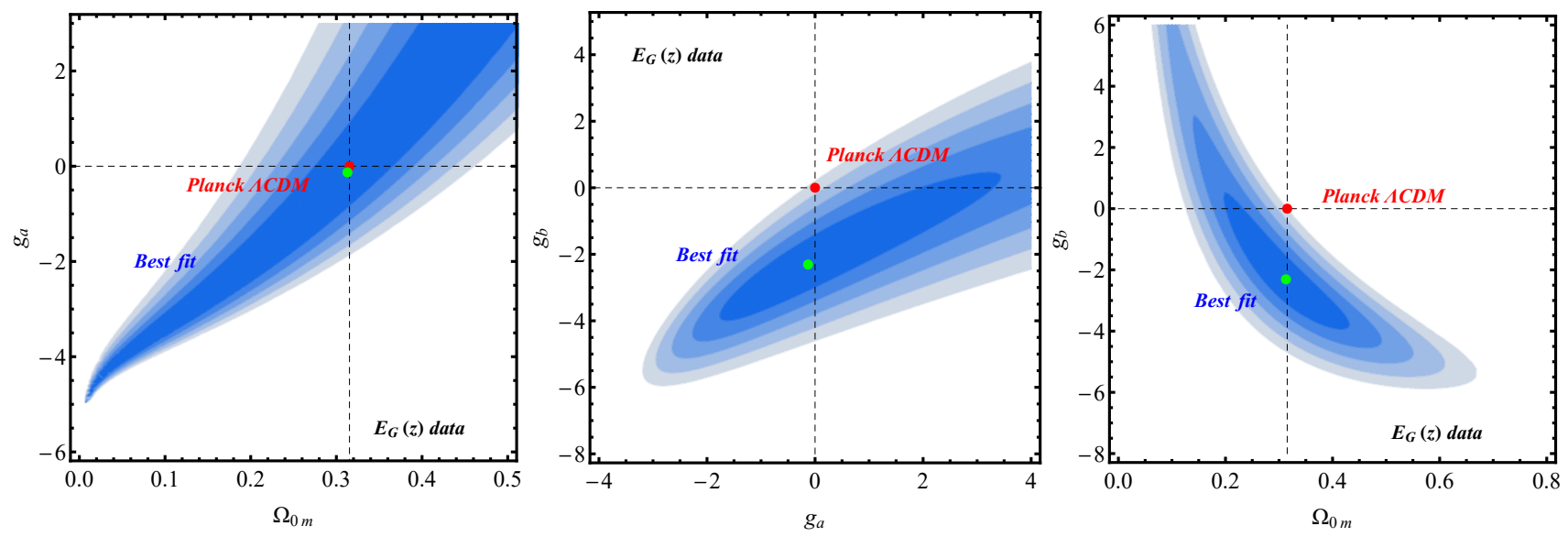

FIG. 4. The three $1 \sigma-5 \sigma$ confidence contours in $2 \mathrm{D}$ projected parameter spaces of the parameter space $\left(\Omega_{0 m}, g_{a}, g_{b}\right)$ in the context of parametrizations Eqs.(2.18) and (2.19) with $n=2, m=2$. The data $E_{G}(z)$ from Table VII of the Appendix B were used. The third parameter in each contour was fixed to the best fit value. The red and green dots describe the Planck18/ $\Lambda$ CDM best fit and the best-fit values from data.

Thus we obtain $\chi_{f \sigma_{8}}^{2}$ as

$$
\chi_{f \sigma_{8}}^{2}\left(\Omega_{0 m}, \sigma_{8}, g_{a}\right)=V_{f \sigma_{8}}^{i} F_{f \sigma_{8}, i j} V_{f \sigma_{8}}^{j}
$$

where $F_{f \sigma_{8}, i j}$ is the Fisher matrix (the inverse of the covariance matrix $C_{f \sigma_{8}, i j}$ of the data) which is assumed to be diagonal with the exception of the $3 \times 3 \mathrm{WiggleZ}$ subspace (see [2] for more details on this compilation).

Similarly, for the construction of $\chi_{E_{G}}^{2}$, we consider the vector

$$
V_{E_{G}}^{i}\left(z_{i}, p\right) \equiv E_{G, i}^{o b s}-E_{G}^{t h}\left(z_{i}, p\right)
$$

where $E_{G, i}^{o b s}$ is the the value of the $i$ th datapoint, with $i=1, \ldots, N_{E_{G}}$ (where $N_{E_{G}}=16$ corresponds to the total number of datapoints of Table VII), while $E_{G}^{t h}\left(z_{i}, p\right)$ is the theoretical prediction (Eq. (2.17)), both at redshift $z_{i}$.
The parameter vector $p$ corresponds to the parameters of Eq. (2.17) with the parametrization (2.18) namely $\Omega_{0 m}$, $g_{a}, g_{b}$.

Thus we obtain $\chi_{E_{G}}^{2}$ as

$$
\chi_{E_{G}}^{2}\left(\Omega_{0 m}, g_{a}, g_{b}\right)=V_{E_{G}}^{i} F_{E_{G}, i j} V_{E_{G}}^{j}
$$

where $F_{E_{G}, i j}$ is the Fisher matrix also assumed to be diagonal.

By minimizing $\chi_{f \sigma_{8}}^{2}, \chi_{E_{G}}^{2}$ separately and combined as $\chi_{t o t}^{2} \equiv \chi_{f \sigma_{8}}^{2}+\chi_{E_{G}}^{2}$ we obtain the constraints on the parameters $\Omega_{0 m}, \sigma_{8}, g_{a}, g_{b}$ shown in Figs. 3, 4 and 5 respectively. Each one of these Figures corresponds to a $2 \mathrm{D}$ projection that goes through the best fit parameter point in parameter space of the full three or four dimensional contour plot in each case. The full number of parameters (three or four) was assumed 

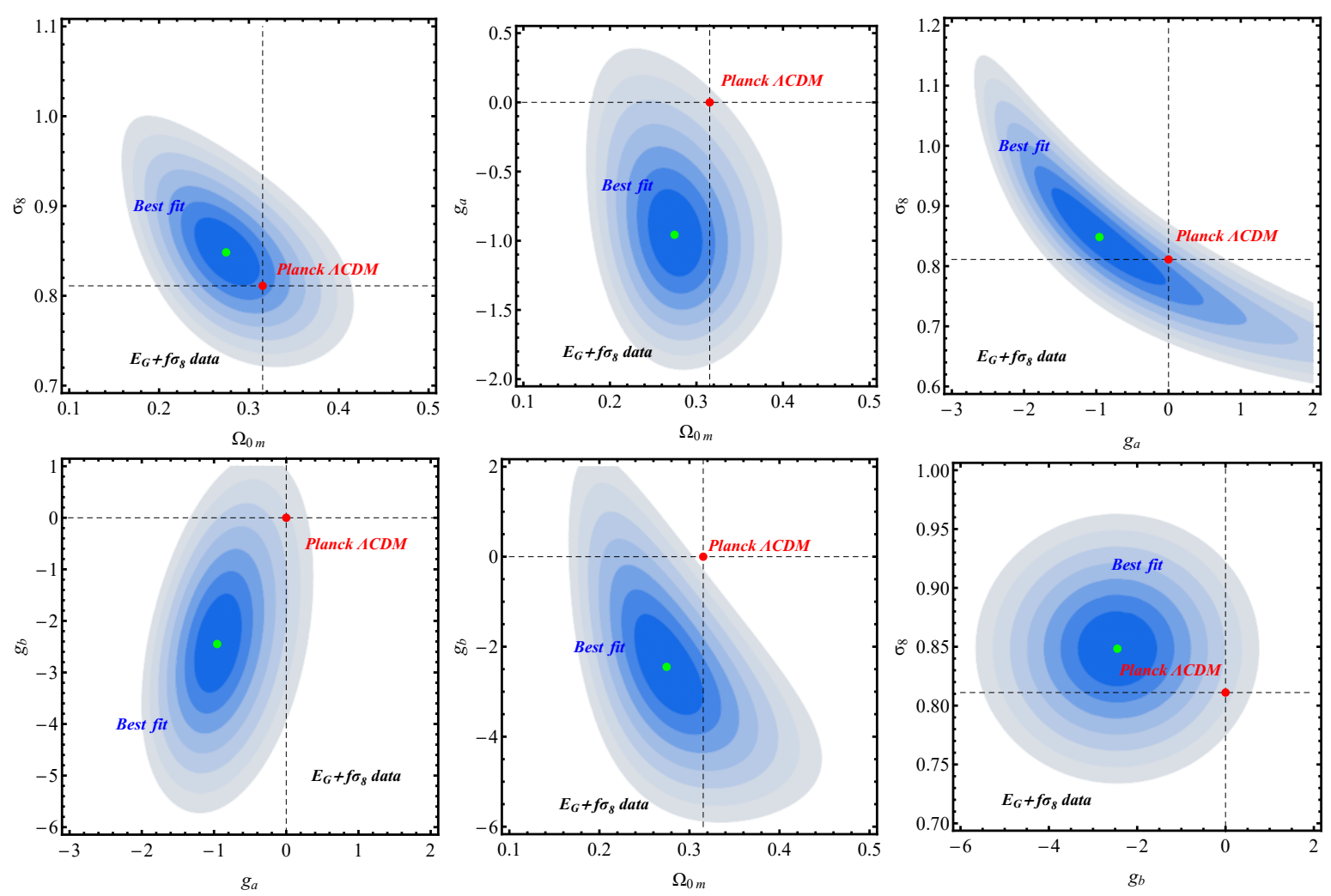

FIG. 5. The six $1 \sigma-7 \sigma$ confidence contours in $2 \mathrm{D}$ projected parameter spaces of the parameter space $\left(\Omega_{0 m}, \sigma_{8}, g_{a}, g_{b}\right)$ in the context of parametrizations Eqs.(2.18)and (2.19) with $n=2$ and $m=2$ including the fiducial correction factor Eq. (3.2). The data $E_{G}(z)$ and $f \sigma_{8}(z)$ from Tables VII and VI of the Appendix B was used. The third and the forth parameter in each contour were fixed to the best fit values. The red and green dots describe the Planck18/ $\Lambda$ CDM best fit and the best-fit values from data.

when constructing the contour 2D projections. Previous studies $[1,2]$ have considered similar 2D projections that go through the Planck/ $\Lambda \mathrm{CDM}$ best fit parameter point in the higher dimensional parameter space. This later choice tends to change somewhat (in most projections it is increased) the apparent tension between the best fit MG parameter values and the best fit Planck/ $\Lambda$ CDM parameters in the $2 \mathrm{D}$ projection parameter subspaces. This 2D tension may be in some cases misleading due to projection effects and thus in Table (III) we stress the tension in the full $3 \mathrm{D}$ or $4 \mathrm{D}$ parameter space.

The tension level between the best fit MG parameter values and the Planck/ $\Lambda \mathrm{CDM}$ best fit parameter values is significant in both the $2 \mathrm{D}$ projection parameter spaces shown in Figs 3, 4 and 5 and in the higher 3D parameter space likelihood surfaces shown in Fig. 6. The best fit parameter values obtained in the context of the datasets considered and the tension levels in both the $2 \mathrm{D}$ projections and in the full $3 \mathrm{D}-4 \mathrm{D}$ parameter spaces are shown in Tables II and III respectively. In these Tables we also show the cases corresponding to fits without including the correction factor (3.2) in the $f \sigma_{8}$ data demonstrating that there is a small change in the best fit parameter values.
The following comments can be made on the results shown in Figs. 3, 4 and 5 and Tables II and III:

- The left part of Table III shows the tension level in the full $3 \mathrm{D}$ or $4 \mathrm{D}$ parameter space. The ten- sion level between Planck/ $\Lambda \mathrm{CDM}$ and best fit MG model parametrizations (2.18) and (2.19) in the context of the $f \sigma_{8}$ data is significant (about $3.5 \sigma$ ) but is is less than the corresponding tension ob- 

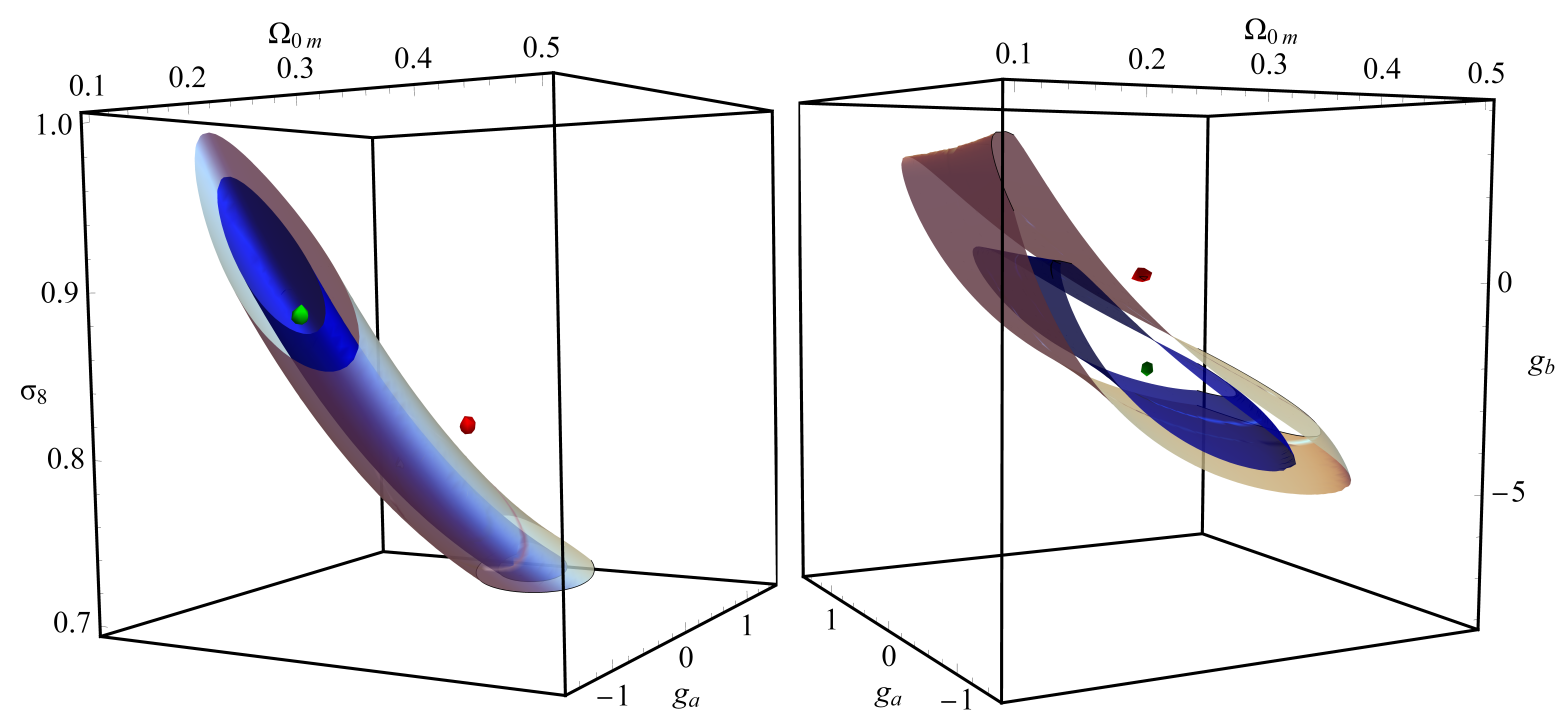

FIG. 6. Left: The $1 \sigma-2 \sigma$ confidence contour of the parameter space $\left(\Omega_{0 m}, \sigma_{8}, g_{a}\right)$ in the context of parametrization Eq. (2.18) with $n=2$ including the fiducial correction factor Eq. (3.2). The RSD data $f \sigma_{8}(z)$ from Table VI of the Appendix B was used. The red and green dots describe the Planck18/ $\Lambda$ CDM best fit and the best-fit values from data. Right: The $1 \sigma-2 \sigma$ confidence contour of the parameter space $\left(\Omega_{0 m}, g_{a}, g_{b}\right)$ in the context of parametrizations Eqs.(2.18) and (2.19) with $n=2$. The data $E_{G}(z)$ from Table VII of the Appendix B was used. The red and green dots describe the Planck18/ $\Lambda$ CDM best fit and the best-fit values from data. The 3D contours include only the surfaces in 3D while the intermediate space is not filled. Thus, the white gaps that appear in the right figure between the surfaces, simply correspond to the white background seen from behind.

\begin{tabular}{|c|c|c|c|c|c|c|}
\hline Param. & Planck18/ $\Lambda \mathrm{CDM}$ & $\begin{array}{c}\text { Dataset } \\
f \sigma_{8}(z) \\
\text { corr. }\end{array}$ & $\begin{array}{c}\text { Dataset } \\
f \sigma_{8}(z) \\
\text { no corr. }\end{array}$ & $\begin{array}{c}\text { Dataset } \\
E_{G}(z)\end{array}$ & $\begin{array}{c}\text { Datasets } \\
f \sigma_{8}(z)+E_{G}(z) \\
\text { corr. }\end{array}$ & $\begin{array}{c}\text { Datasets } \\
f \sigma_{8}(z)+E_{G}(z) \\
\text { no corr. }\end{array}$ \\
\hline$\Omega_{0 m}$ & $0.3153 \pm 0.0073$ & $0.272 \pm 0.019$ & $0.263 \pm 0.015$ & $0.313 \pm 0.024$ & $0.275 \pm 0.015$ & $0.264 \pm 0.012$ \\
\hline$\sigma_{8}$ & $0.8111 \pm 0.0060$ & $0.886 \pm 0.015$ & $0.90 \pm 0.016$ & & $0.848 \pm 0.015$ & $0.879 \pm 0.015$ \\
\hline$g_{a}$ & 0 & $-1.306 \pm 0.140$ & $-1.331 \pm 0.138$ & $-0.129 \pm 0.490$ & $-0.957 \pm 0.144$ & $-1.115 \pm 0.137$ \\
\hline$g_{b}$ & 0 & & & $-2.308 \pm 0.423$ & $-2.448 \pm 0.414$ & $-2.422 \pm 0.416$ \\
\hline
\end{tabular}

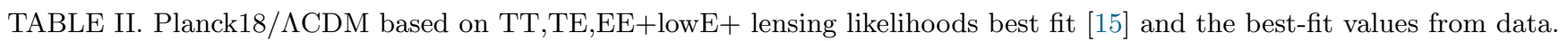

tained using the $E_{G}$ statistic data (more than $4 \sigma$ ). In fact for the combined $f \sigma_{8}+E_{g}$ dataset the tension level increases to close to $6 \sigma$ ! This significant tension level comes independently from both the $f \sigma_{8}$ and $E_{G}$ data and hints towards weaker gravity ( $\mu$ and $\Sigma$ lower than 1) compared to the predictions of GR at low $z$. We stress however that this extreme level of tension is partly due to correlations among the considered datapoints which necessarily exist in our compilations.

The trend for weaker gravity at low redshifts is also evident in Fig. 7 which shows the best fit form of $\mu(a)$
- The weaker than expected gravitational growth indicated by the data is expressed as both a lower best fit $\Omega_{0 m}$ than expected from $\Lambda$ CDM and as negative best fit values for the gravitational strength evolution parameters $g_{a}$ and $g_{b}$ (see e.g. Fig. 5).

- Ignoring the fiducial model correction factor of Eq. (3.2) in most cases tends to slightly increase the tension level (compare e.g. the last two lines of Table III). Thus the consideration of this correction in our analysis is a conservative approach.

and $\Sigma(a)$ in the context of each dataset.

Also, the likelihood contours in the $\sigma_{8}-\Omega_{m}$ parameter 


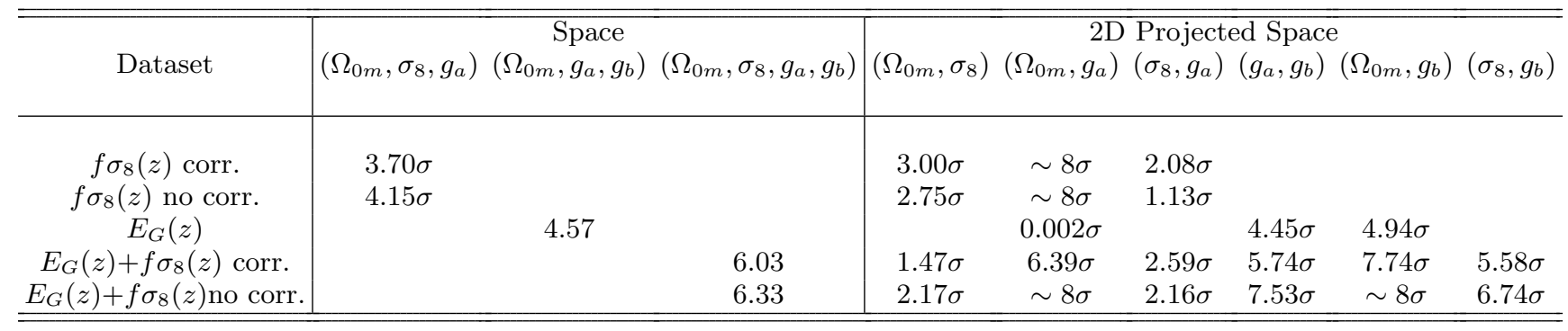

TABLE III. Sigma differences of the best fit contours from Planck18/ $\Lambda$ CDM.
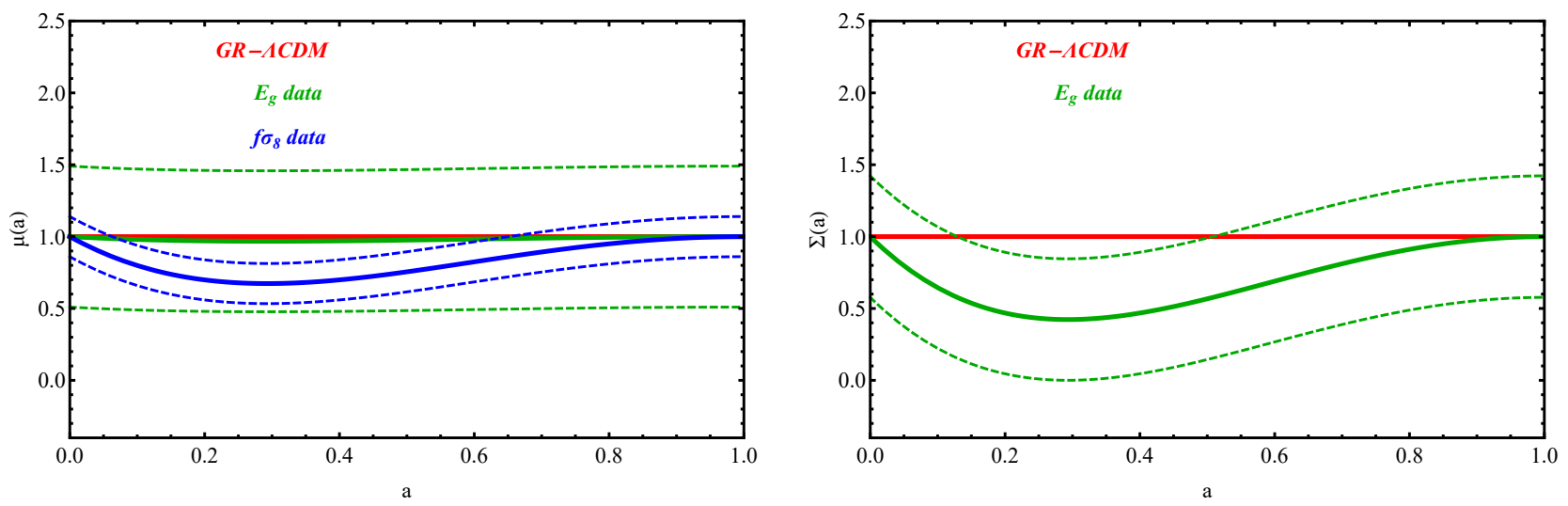

FIG. 7. Evolution of $\mu$ and $\Sigma$ as functions of the scale factor $a$ considering the best fit values for $g_{a}$ and $g_{b}$ in the context of parametrizations Eqs.(2.18) and (2.19) with $n=2, m=2$. The data $E_{G}(z)$ and $f \sigma_{8}(z)$ from Tables VII and VI of the Appendix B was used. The dashed curves correspond to $1 \sigma$ deviations of the parameters $\mu$ and $\Sigma$. The red lines correspond to the GR- $\Lambda$ CDM model.

space obtained using the growth data in the presence of the MG parameter $g_{a}$ and in the context of GR $\left(g_{a}=0\right)$ are shown in Fig. 8. We have considered both the case of a marginalized MG parameter value and the case of setting $g_{a}$ to its best fit value. Clearly the tension level between the best fit parameter values and Planck/ $\Lambda$ CDM decreases significantly in the presence of the MG parameter $g_{a}$.

The introduction of additional parameters of any type would in general widen the likelihood contours and thus reduce the tension between growth data and geometric/CMB data. In general a faster expansion rate $(w<-1)$ would tend to reduce the growth rate of perturbations in agreement with dynamical observables. However, geometric observables (SnIa, BAO etc.) do not allow significant deviations of the expansion rate from $\Lambda$ CDM. Thus the most efficient way to produce a weaker growth of perturbations is the introduction of evolution of the MG parameters $\mu$ and $\Sigma$. In Fig 9 we have demonstrated this effect by fixing $g_{a}=0, g_{b}=0$ and constructing the $\sigma_{8}-\Omega_{m}$ contours with $w=-1$ and $w$ free to vary in a range $([-1.5,-0.5])$ consistent with geometric probes. The reduction of the tension in this case is significantly smaller compared to the introduction of MG degrees of freedom.

\section{III.2. Scale Dependent Data Compilations}

Scale dependent parametrizations for $\mu$ and $\eta$ can describe a large class of MG models [86, 106]. For example a scale dependent class of parametrizations predicted by scalar-tensor theories for $\mu$ and $\eta$ is of the form [120, 121]

$$
\begin{aligned}
& \mu(a, k)=1+f_{1}(a) \frac{1+c_{1}(\lambda H / k)^{2}}{1+(\lambda H / k)^{2}} \\
& \eta(a, k)=1+f_{2}(a) \frac{1+c_{2}(\lambda H / k)^{2}}{1+(\lambda H /)^{2}}
\end{aligned}
$$

where $f_{1}$ and $f_{2}$ are properly chosen functions that depend on the scale factor. Thus a physically motivated scale dependent generalization of the parametrizations (2.18) and (2.19) for $\mu$ and $\Sigma$ may be written as

$$
\begin{aligned}
& \mu(R, z)=1+\left[g_{a}\left(\frac{z}{1+z}\right)^{n}-g_{a}\left(\frac{z}{1+z}\right)^{2 n}\right] \frac{1+s_{a}(\lambda H R)^{2}}{1+(\lambda H R)^{2}} \\
& (3.8) \\
& \Sigma(R, z)=1+\left[g_{b}\left(\frac{z}{1+z}\right)^{m}-g_{b}\left(\frac{z}{1+z}\right)^{2 m}\right] \frac{1+s_{b}(\lambda H R)^{2}}{1+(\lambda H R)^{2}}
\end{aligned}
$$



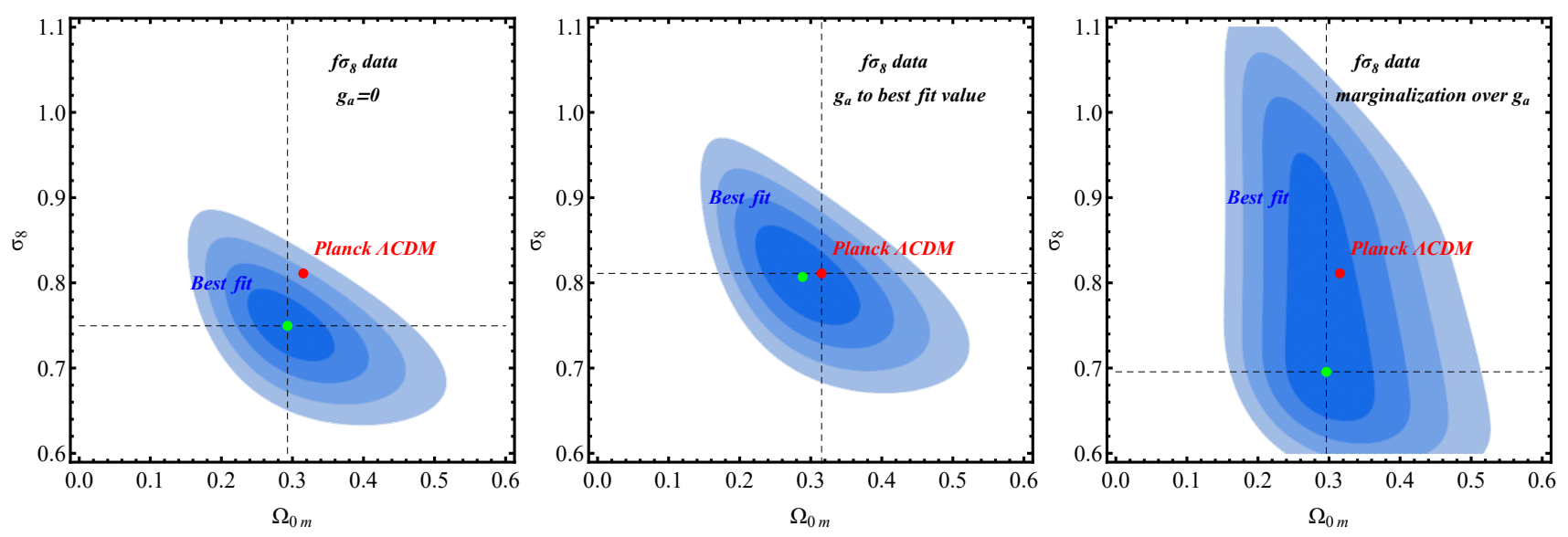

FIG. 8. The confidence contours of the parameter space $\left(\sigma_{8}-\Omega_{m}\right)$ in the context of GR (left panel) and in the presence of the MG parameter $g_{a}$ (fixing $w=-1$ ) in the context of parametrization Eq.(2.18) with $n=2$. We have considered both the case of a marginalized MG parameter value (right panel) and the case of setting $g_{a}$ to its best fit value (middle panel). The red and green dots describe the Planck18/ $\Lambda \mathrm{CDM}$ best fit and the best-fit values from data. The $f \sigma_{8}(z)$ data compilations of datapoints with less correlation from Table VI of the Appendix B was used. Notice the reduction of tension between the growth data best fit and the Planck/ $\Lambda$ CDM parameter values when the MG degree of freedom is introduced.

where $s_{a}, s_{b}$ and $\lambda$ are parameters to be determined from a proper scale dependent dataset. Such a scale dependent data compilation for the statistic $E_{G}$ in two redshift ranges is shown in Fig. 10 and in Tables VIII and IX for low and high $z$ respectively in the Appendix B. The analysis of this compilation may be performed in the context of the scale dependent parametrizations (3.8) and (3.9). Clearly as shown in Fig. 10, for both low and high $z$ the scale independent MG parametrizations of Eqs.(2.18) and (2.19) at $z=0.3$ and at $z=0.7$, lead to a best fit value of $E_{G}$ that is lower compared to the Planck/ $\Lambda \mathrm{CDM}$ prediction. The full scale dependent analysis leads to similar levels of tension as those indicated in Table III for the scale independent case and will be presented in detail elsewhere.

\section{CONCLUSIONS-DISCUSSION}

We have used up to date compilations of $E_{G}$ and $f \sigma_{8}$ data (Tables VI and VII) based on WL and RSD observations to obtain updated estimates of the tension between the Planck/ $\Lambda \mathrm{CDM}$ best fit parameter values and the best fit parameter values obtained in the context of an effective MG gravity model allowing for properly parametrized evolution of the growth and lensing gravitational constants $\mu$ and $\Sigma$. The scale independent parametrizations (Eqs.(2.18), (2.19)) of $\mu$ and $\Sigma$ depend on the parameters $g_{a}$ and $g_{b}$ respectively and are by construction consistent with GR at early times and at present as indicated by nucleosynthesis and solar system constraints assuming no screening is present. We have assumed a flat $\Lambda \mathrm{CDM}$ expansion background and we thus fit the parameters $\left(\Omega_{0 m}, \sigma_{8}, g_{a}, g_{b}\right)$.
We find that the $E_{G}$ data amplify the previously well known indications for low $\Omega_{0 m}$ and/or weaker gravity $(\mu<1)$ at low $z$ and favor weaker gravity for both the growth and the lensing gravitational constants $(\mu<1$ and $\Sigma<1)$. The tension level between the Planck/ $\Lambda \mathrm{CDM}$ parameter values $\left(\Omega_{0 m}, \sigma_{8}, g_{a}, g_{b}\right)=(0.31,0.81,0,0)$ and the best fit parameter values obtained using the combined $E_{G}+f \sigma_{8}$ dataset $\left(\Omega_{0 m}, \sigma_{8}, g_{a}, g_{b}\right)=(0.28,0.85,-0.96,-2.45)$ is $6 \sigma$ which is significantly larger compared to the tension obtained when only the $f \sigma_{8}$ dataset is used ( $3.7 \sigma$ as shown in Table III). Even though the absolute magnitude of the derived tension is overestimated due to the correlations among the datapoints the amplified trend for weaker gravity at low $z$ is clearly indicated by both the $f \sigma_{8}$ and $E_{G}$ data compilations and appears to be stronger for the case of the $E_{G}$ data.

If this trend has some physical origin and is not due only to data systematics or physical effects in the context of GR, there are significant implications for theoretical models. In particular $f(R)$ theories generically predict stronger gravity at low $z$ compared to its present time[122] (thus the prediction is $\mu(z)>1, g_{a}>0$ ) and therefore if the identified tension has physical origin this can not be attributed to an $f(R)$ MG gravity theory for any expansion background. Similarly minimal scalar tensor theories [3, 122], Horndeski theories [123, 124] and beyond Horndeski Gleyzes-Langlois-Piazza-Vernizzi (GLPV) theories [125] can only produce weaker gravity at low $z$ under very specific and in some cases unnatural conditions[52]. For example minimal scalar-tensor theories would require the existence of a phantom comsological background expansion (equation of state parameter $w<-1)[3,122]$. In fact, a very large class of MG 

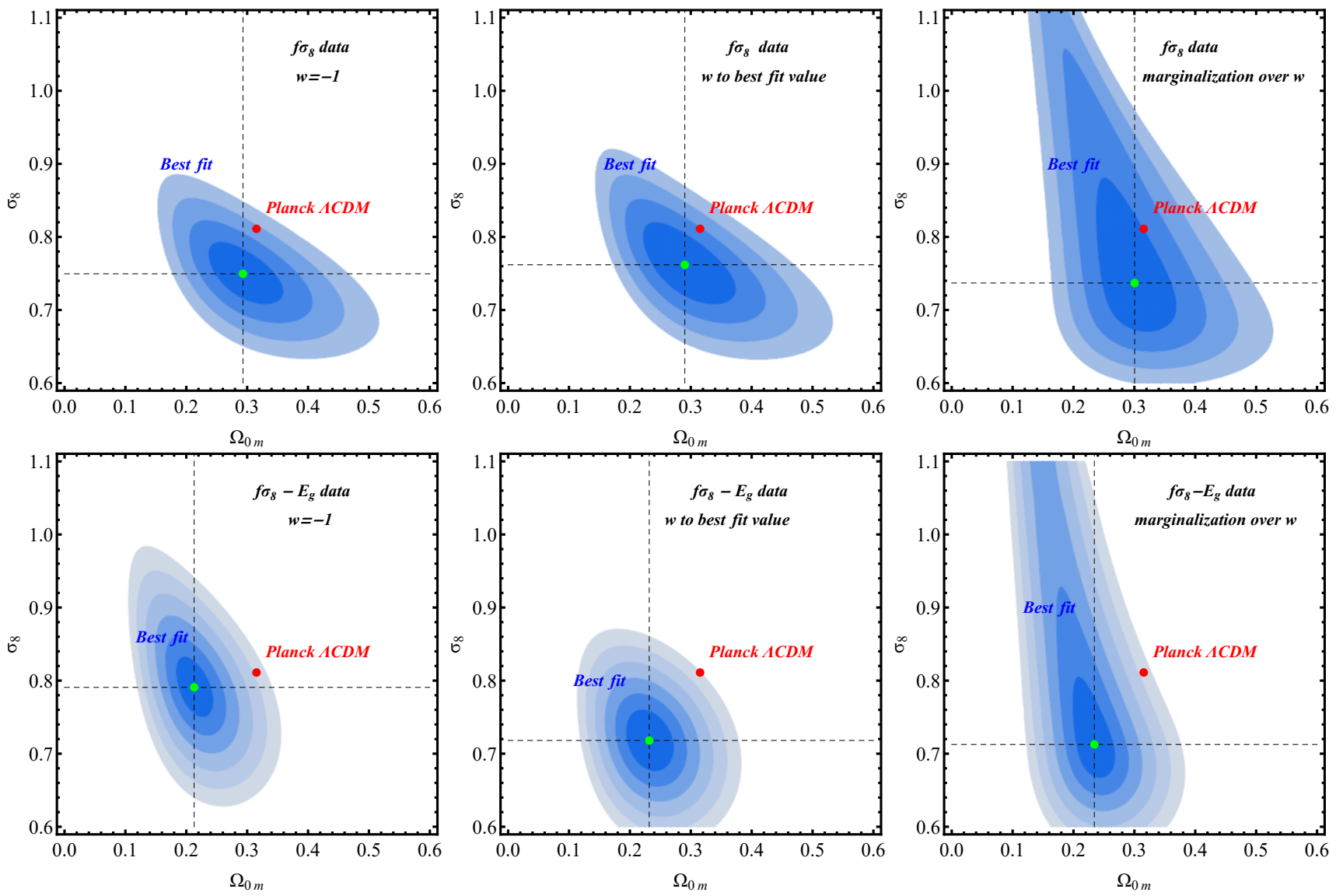

FIG. 9. The confidence contours of the parameter space $\left(\sigma_{8}-\Omega_{m}\right)$ in the context of GR (left panels) and in the presence of the $w$ parameter (fixing $g_{a}=0$ and $\left.g_{b}=0\right)$. We have considered both the case of a marginalized $w([-1.5,-0.5])$ parameter value (right panels) and the case of setting $w$ to its best fit value $\left(-0.94\right.$ and -1.29 from $f \sigma_{8}(z)$ and $f \sigma_{8}(z)+E_{G}(z)$ data respectively) (middle panels). The red and green dots describe the Planck18/ $\Lambda$ CDM best fit and the best-fit values from data. The $E_{G}(z)$ and $f \sigma_{8}(z)$ data compilations of datapoints with less correlation from Tables VII and VI of the Appendix B was used. Notice that the reduction of tension between the best fit parameter values and Planck/ $\Lambda$ CDM is less efficient when the $w$ degree of freedom (modified background expansion rate) is introduced compared to the MG degree of freedom $g_{a}$ shown in Fig. 8 .
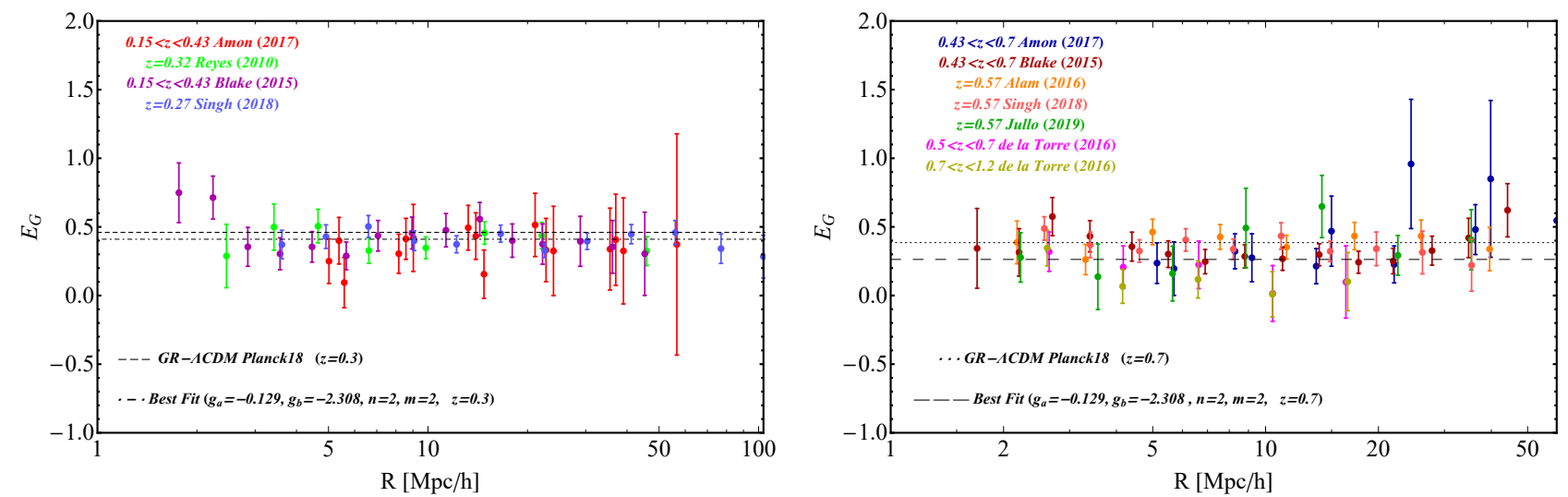

FIG. 10. Measurements of $E_{G}$ as a function of scale $R$ in the range $0.15<z<0.43$ (left panel) and $0.43<z<1.2$ (right panel). The data $E_{G}(R)$ from Tables VIII and IX of the Appendix B was used. The dashed black line shows the Planck18/ $\Lambda$ CDM prediction at $z=0.3$, the dotted black line shows the Planck18/ $\Lambda$ CDM prediction at $z=0.7$, while the dotdashed black line and the large dashed black line shows the best fit of the $E_{G}$ in the context of parametrizations Eqs.(2.18) and (2.19) at $z=0.3$ and at $z=0.7$ respectively. 


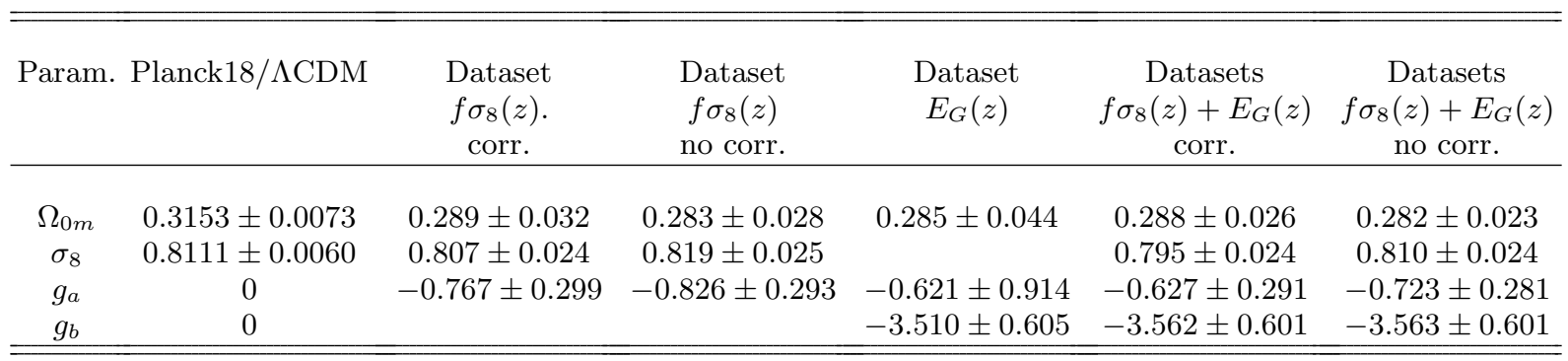

TABLE IV. Planck18/ $\Lambda$ CDM based on TT,TE,EE+lowE+ lensing likelihoods best fit [15] and the best-fit values from data compilation of datapoints with less correlation.

models, the scalar-tensor Horndeski models, are not consistent with the observational indications of weakening gravity. In fact as stated in Ref. [126] (p. 12), $\mu$ for stable Horndeski models is always larger than, or equal to, 1 so that matter perturbations in viable Horndeski models always grow faster than the corresponding GR models with the same backgrounds. Thus these MG models (which include $f(R)$ gravities) are unable to account for the weakening and would provide a worse fit than GR to the $f \sigma_{8} / E_{G}$ data. The search for MG models that can account for the observed indications for weakening gravity is thus an interesting extension of the present analysis.

A partial cause of the $E_{G}$ data tension with Planck $/ \Lambda C D M$ is lensing magnification. As shown in $[127,128]$ the effects of lensing magnification modify the galaxy-galaxy lensing correlations as well as galaxygalaxy correlations and as a consequence introduce systematic errors in the estimate of $E_{G}$ while making it bias dependent. The effect is small for redshifts smaller than 1 (about $5-10 \%$ ) but it can become as large as $20-40 \%$ for redshifts $z \simeq 1.5$. Thus, this systematic contribution can be relevant already for Dark Energy Survey (DES) [38, 129-132] and certainly for higher redshift surveys. However, the magnitude of lensing contribution at the redshifts of the data compilation we are using $(z<1)$ is not large enough to significantly reduce the identified tension which exists even at the level of the RSD data alone. The systematic effect discussed in [127, 128] is important especially for upcoming surveys like Euclid [133] which probe higher redshifts even though even in that case it may not be large enough to be the only source the observed tension. An interesting feature of our compilation is the scale dependence the $E_{G}(R, z)$ data. This may be used to probe the parameters of scale dependent MG $\mu$ and $\Sigma$ parametrizations which are well motivated physically. We plan to present such constraints elsewhere using upcoming and more extensive data able to constrain the required larger parameter space that appears in scale dependent $\mu$ and $\Sigma$ parametrizations. A key question to address is whether the addition of scale dependence in the parametrizations can improve significantly the overall fit. No such indications are currently known [120] but this may well change using more extensive and accurate scale dependent $E_{G}$ and $f \sigma_{8}$ data.

The introduction of the MG parameters $\mu$ and $\Sigma$ along with the variation of the parameters $\Omega_{m}$ and $\sigma_{8}$ leads to a model (MG- $\Lambda \mathrm{CDM}$ ) that is a much better fit to the growth $f \sigma_{8}$ and $E_{G}$ data than the Planck/ $\Lambda \mathrm{CDM}$ model in the context of GR. We have called this effect a 'tension' between the new best fit parameter values $(\mathrm{MG}-\Lambda \mathrm{CDM})$ and the GR-Planck/ $\Lambda \mathrm{CDM}$ parameter values (from Planck18 fit) which are $5-6 \sigma$ away from the new best fit parameter values. On the other hand, the MG parameters do not seem to change significantly the fit of the Planck data as indicated in Ref. [4] and in Planck18 [15] which indicate that pure CMB data appear to favor GR. Thus, the particular parametrization we have used does not seem to significantly reduce the tension between $\mathrm{CMB}$ and growth/weak lensing data since MG gravity appears to be favored by growth/weak lensing but not by the CMB. This is an issue we plan to investigate in more detail in the future by considering e.g. different MG parametrizations for the evolution of the $\mu$ and $\Sigma$ parameters that will not only improve the fit to the $f \sigma_{8} / E_{G}$ data but also improve the fit to the CMB data where some tensions are already evident (e.g. the lensing anomaly discussed in Planck18 [15]).

Supplemental Material: The Mathematica file used for the numerical analysis and for construction of the figures can be found in [134].

\section{ACKNOWLEDGEMENTS}

We thank Savvas Nesseris and Ruth Durrer for useful comments. This article has benefited from COST Action CA15117 (CANTATA), supported by COST (European Cooperation in Science and Technology). 


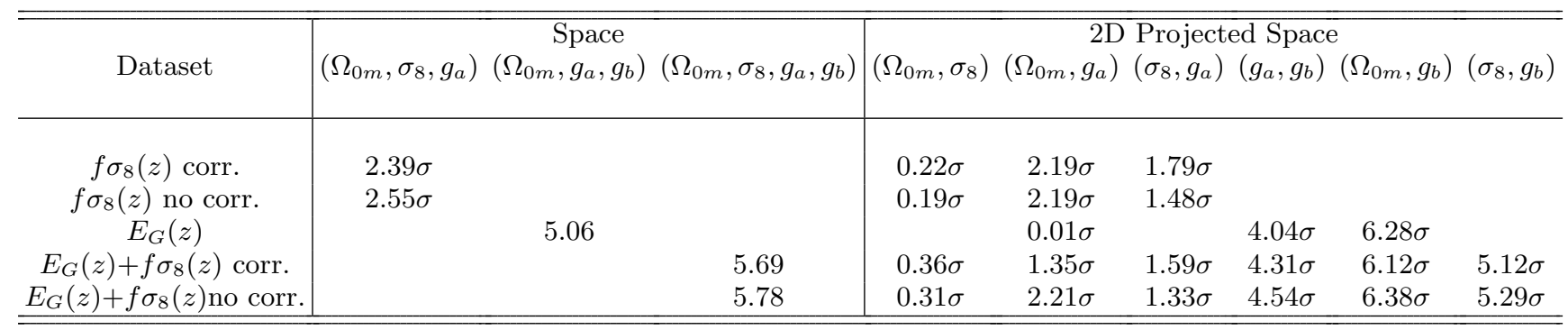

TABLE V. Sigma differences of the best fit contours from Planck18/ $\Lambda$ CDM. The $E_{G}(z)$ and $f \sigma_{8}(z)$ data compilations of datapoints with less correlation from Tables VII and VI of the Appendix B was used.

\section{Appendix A: ANALYSIS OF SUBSETS OF DATAPOINTS WITH LESS CORRELATION}

In this Appendix we present the results of the statistical analysis of the $f \sigma_{8}(z)$ and $E_{G}(z)$ data compilations of datapoints with less correlation. These subsets of the data are indicated with bold font in the index of the Tables VI and VII of the Appendix B. Using these subsets of the data and repeating our analysis we obtain the best fit parameter values and the tension levels in both the $2 \mathrm{D}$ projections and in the full $3 \mathrm{D}-4 \mathrm{D}$ parameter spaces as shown in Tables IV and V respectively.

These results indicate that even though the tension level for the combined $\left(E_{G}+f \sigma_{8}\right)$ reduces somewhat (from $6 \sigma$ to about $5.5 \sigma$ ) it remains high enough to cause concerns for the self consistency of the Planck/ $\Lambda \mathrm{CDM}$ model and indications for the presence of weakening gravity.

\section{Appendix B: DATA USED IN THE ANALYSIS}

In this appendix we present the data used in the analysis.

TABLE VI: The $f \sigma_{8}$ updated data compilation of Ref. [2] used in the present analysis. The subset of the datapoints with less correlation is indicated with bold font in the index.

\begin{tabular}{|c|c|c|c|c|c|c|}
\hline Index & Dataset & $z$ & $f \sigma_{8}(z)$ & Refs. & Year & Fiducial Cosmology \\
\hline 1 & SDSS-LRG & 0.35 & $0.440 \pm 0.050$ & [135] & 30 October 2006 & $\overline{\left(\Omega_{m}, \Omega_{K}, \sigma_{8}\right)=(0.25,0,0.756)[136]}$ \\
\hline 2 & VVDS & 0.77 & $0.490 \pm 0.18$ & {$[135]$} & 6 October 2009 & $\left(\Omega_{m}, \Omega_{K}, \sigma_{8}\right)=(0.25,0,0.78)$ \\
\hline 3 & 2dFGRS & 0.17 & $0.510 \pm 0.060$ & {$[135]$} & 6 October 2009 & $\left(\Omega_{m}, \Omega_{K}\right)=(0.3,0,0.9)$ \\
\hline 4 & 2MRS & 0.02 & $0.314 \pm 0.048$ & [137], [138] & 13 November 2010 & $\left(\Omega_{m}, \Omega_{K}, \sigma_{8}\right)=(0.266,0,0.65)$ \\
\hline 5 & SnIa+IRAS & 0.02 & $0.398 \pm 0.065$ & [139], [138] & 20 October 2011 & $\left(\Omega_{m}, \Omega_{K}, \sigma_{8}\right)=(0.3,0,0.814)$ \\
\hline 6 & SDSS-LRG-200 & 0.25 & $0.3512 \pm 0.0583$ & [140] & 9 December 2011 & $\left(\Omega_{m}, \Omega_{K}, \sigma_{8}\right)=(0.276,0,0.8)$ \\
\hline 7 & SDSS-LRG-200 & 0.37 & $0.4602 \pm 0.0378$ & {$[140]$} & 9 December 2011 & \\
\hline 8 & SDSS-LRG-60 & 0.25 & $0.3665 \pm 0.0601$ & {$[140]$} & 9 December 2011 & $\left(\Omega_{m}, \Omega_{K}, \sigma_{8}\right)=(0.276,0,0.8)$ \\
\hline 9 & SDSS-LRG-60 & 0.37 & $0.4031 \pm 0.0586$ & {$[140]$} & 9 December 2011 & \\
\hline 10 & WiggleZ & 0.44 & $0.413 \pm 0.080$ & {$[141]$} & 12 June 2012 & $\left(\Omega_{m}, h, \sigma_{8}\right)=(0.27,0.71,0.8)$ \\
\hline 11 & WiggleZ & 0.60 & $0.390 \pm 0.063$ & {$[141]$} & 12 June 2012 & \\
\hline 12 & WiggleZ & 0.73 & $0.437 \pm 0.072$ & {$[141]$} & 12 June 2012 & \\
\hline 13 & $6 \mathrm{dFGS}$ & 0.067 & $0.423 \pm 0.055$ & {$[84]$} & 4 July 2012 & $\left(\Omega_{m}, \Omega_{K}, \sigma_{8}\right)=(0.27,0,0.76)$ \\
\hline 14 & SDSS-BOSS & 0.30 & $0.407 \pm 0.055$ & [142] & 11 August 2012 & $\left(\Omega_{m}, \Omega_{K}, \sigma_{8}\right)=(0.25,0,0.804)$ \\
\hline 15 & SDSS-BOSS & 0.40 & $0.419 \pm 0.041$ & {$[142]$} & 11 August 2012 & \\
\hline 16 & SDSS-BOSS & 0.50 & $0.427 \pm 0.043$ & {$[142]$} & 11 August 2012 & \\
\hline 17 & SDSS-BOSS & 0.60 & $0.433 \pm 0.067$ & {$[142]$} & 11 August 2012 & \\
\hline 18 & VIPERS & 0.80 & $0.470 \pm 0.080$ & {$[143]$} & 9 July 2013 & $\left(\Omega_{m}, \Omega_{K}, \sigma_{8}\right)=(0.25,0,0.82)$ \\
\hline 19 & SDSS-DR7-LRG & 0.35 & $0.429 \pm 0.089$ & {$[144]$} & 8 August 2013 & $\left(\Omega_{m}, \Omega_{K}, \sigma_{8}\right)=(0.25,0,0.809)[145]$ \\
\hline 20 & GAMA & 0.18 & $0.360 \pm 0.090$ & {$[146]$} & 22 September 2013 & $\left(\Omega_{m}, \Omega_{K}, \sigma_{8}\right)=(0.27,0,0.8)$ \\
\hline 21 & GAMA & 0.38 & $0.440 \pm 0.060$ & {$[146]$} & 22 September 2013 & \\
\hline 22 & BOSS-LOWZ & 0.32 & $0.384 \pm 0.095$ & {$[147]$} & 17 December 2013 & $\left(\Omega_{m}, \Omega_{K}, \sigma_{8}\right)=(0.274,0,0.8)$ \\
\hline 23 & SDSS DR10 and DR11 & 0.32 & $0.48 \pm 0.10$ & {$[147]$} & 17 December 2013 & $\left(\Omega_{m}, \Omega_{K}, \sigma_{8}\right)=(0.274,0,0.8)[148]$ \\
\hline
\end{tabular}




\begin{tabular}{|c|c|c|c|c|c|c|}
\hline 24 & SDSS DR10 and DR11 & 0.57 & $0.417 \pm 0.045$ & [147] & 17 December 2013 & \\
\hline 25 & SDSS-MGS & 0.15 & $0.490 \pm 0.145$ & [149] & 30 January 2015 & $\left(\Omega_{m}, h, \sigma_{8}\right)=(0.31,0.67,0.83)$ \\
\hline 26 & SDSS-veloc & 0.10 & $0.370 \pm 0.130$ & [150] & 16 June 2015 & $\left(\Omega_{m}, \Omega_{K}, \sigma_{8}\right)=(0.3,0,0.89)[151]$ \\
\hline 27 & FastSound & 1.40 & $0.482 \pm 0.116$ & {$[152]$} & 25 November 2015 & $\left(\Omega_{m}, \Omega_{K}, \sigma_{8}\right)=(0.27,0,0.82)[153]$ \\
\hline 28 & SDSS-CMASS & 0.59 & $0.488 \pm 0.060$ & [154] & 8 July 2016 & $\left(\Omega_{m}, h, \sigma_{8}\right)=(0.307115,0.6777,0.8288)$ \\
\hline 29 & BOSS DR12 & 0.38 & $0.497 \pm 0.045$ & {$[155]$} & 11 July 2016 & $\left(\Omega_{m}, \Omega_{K}, \sigma_{8}\right)=(0.31,0,0.8)$ \\
\hline 30 & BOSS DR12 & 0.51 & $0.458 \pm 0.038$ & {$[155]$} & 11 July 2016 & \\
\hline 31 & BOSS DR12 & 0.61 & $0.436 \pm 0.034$ & {$[155]$} & 11 July 2016 & \\
\hline 32 & BOSS DR12 & 0.38 & $0.477 \pm 0.051$ & {$[156]$} & 11 July 2016 & $\left(\Omega_{m}, h, \sigma_{8}\right)=(0.31,0.676,0.8)$ \\
\hline 33 & BOSS DR12 & 0.51 & $0.453 \pm 0.050$ & {$[156]$} & 11 July 2016 & \\
\hline 34 & BOSS DR12 & 0.61 & $0.410 \pm 0.044$ & {$[156]$} & 11 July 2016 & \\
\hline 35 & VIPERS v7 & 0.76 & $0.440 \pm 0.040$ & {$[157]$} & 26 October 2016 & $\left(\Omega_{m}, \sigma_{8}\right)=(0.308,0.8149)$ \\
\hline 36 & VIPERS v7 & 1.05 & $0.280 \pm 0.080$ & {$[157]$} & 26 October 2016 & \\
\hline 37 & BOSS LOWZ & 0.32 & $0.427 \pm 0.056$ & [158] & 26 October 2016 & $\left(\Omega_{m}, \Omega_{K}, \sigma_{8}\right)=(0.31,0,0.8475)$ \\
\hline 38 & BOSS CMASS & 0.57 & $0.426 \pm 0.029$ & {$[158]$} & 26 October 2016 & \\
\hline 39 & VIPERS & 0.727 & $0.296 \pm 0.0765$ & [159] & 21 November 2016 & $\left(\Omega_{m}, \Omega_{K}, \sigma_{8}\right)=(0.31,0,0.7)$ \\
\hline 40 & 6dFGS+SnIa & 0.02 & $0.428 \pm 0.0465$ & {$[160]$} & 29 November 2016 & $\left(\Omega_{m}, h, \sigma_{8}\right)=(0.3,0.683,0.8)$ \\
\hline 41 & VIPERS PDR2 & 0.60 & $0.550 \pm 0.120$ & [161] & 16 December 2016 & $\left(\Omega_{m}, \Omega_{b}, \sigma_{8}\right)=(0.3,0.045,0.823)$ \\
\hline 42 & VIPERS PDR2 & 0.86 & $0.400 \pm 0.110$ & {$[161]$} & 16 December 2016 & \\
\hline 43 & SDSS DR13 & 0.1 & $0.48 \pm 0.16$ & [162] & 22 December 2016 & $\left(\Omega_{m}, \sigma_{8}\right)=(0.25,0.89)[151]$ \\
\hline 44 & $2 \mathrm{MTF}$ & 0.001 & $0.505 \pm 0.085$ & {$[163]$} & 16 June 2017 & $\left(\Omega_{m}, \sigma_{8}\right)=(0.3121,0.815)$ \\
\hline 45 & VIPERS PDR2 & 0.85 & $0.45 \pm 0.11$ & [164] & 31 July 2017 & $\left(\Omega_{b}, \Omega_{m}, h\right)=(0.045,0.30,0.8)$ \\
\hline 46 & BOSS DR12 & 0.31 & $0.384 \pm 0.083$ & {$[165]$} & 15 September 2017 & $\left(\Omega_{m}, h, \sigma_{8}\right)=(0.307,0.6777,0.8288)$ \\
\hline 47 & BOSS DR12 & 0.36 & $0.409 \pm 0.098$ & {$[165]$} & 15 September 2017 & \\
\hline 48 & BOSS DR12 & 0.40 & $0.461 \pm 0.086$ & {$[165]$} & 15 September 2017 & \\
\hline 49 & BOSS DR12 & 0.44 & $0.426 \pm 0.062$ & {$[165]$} & 15 September 2017 & \\
\hline 50 & BOSS DR12 & 0.48 & $0.458 \pm 0.063$ & {$[165]$} & 15 September 2017 & \\
\hline 51 & BOSS DR12 & 0.52 & $0.483 \pm 0.075$ & [165] & 15 September 2017 & \\
\hline 52 & BOSS DR12 & 0.56 & $0.472 \pm 0.063$ & {$[165]$} & 15 September 2017 & \\
\hline 53 & BOSS DR12 & 0.59 & $0.452 \pm 0.061$ & {$[165]$} & 15 September 2017 & \\
\hline 54 & BOSS DR12 & 0.64 & $0.379 \pm 0.054$ & {$[165]$} & 15 September 2017 & \\
\hline 55 & SDSS DR7 & 0.1 & $0.376 \pm 0.038$ & {$[166]$} & 12 December 2017 & $\left(\Omega_{m}, \Omega_{b}, \sigma_{8}\right)=(0.282,0.046,0.817)$ \\
\hline 56 & SDSS-IV & 1.52 & $0.420 \pm 0.076$ & {$[167]$} & 8 January 2018 & $\left(\Omega_{m}, \Omega_{b} h^{2}, \sigma_{8}\right)=(0.26479,0.02258,0.8)$ \\
\hline 57 & SDSS-IV & 1.52 & $0.396 \pm 0.079$ & [168] & 8 January 2018 & $\left(\Omega_{m}, \Omega_{b} h^{2}, \sigma_{8}\right)=(0.31,0.022,0.8225)$ \\
\hline 58 & SDSS-IV & 0.978 & $0.379 \pm 0.176$ & [169] & 9 January 2018 & $\left(\Omega_{m}, \sigma_{8}\right)=(0.31,0.8)$ \\
\hline 59 & SDSS-IV & 1.23 & $0.385 \pm 0.099$ & [169] & 9 January 2018 & \\
\hline 60 & SDSS-IV & 1.526 & $0.342 \pm 0.070$ & [169] & 9 January 2018 & \\
\hline 61 & SDSS-IV & 1.944 & $0.364 \pm 0.106$ & [169] & 9 January 2018 & \\
\hline 62 & VIPERS PDR2 & 0.60 & $0.49 \pm 0.12$ & {$[170]$} & 6 June 2018 & $\left(\Omega_{b}, \Omega_{m}, h, \sigma_{8}\right)=(0.045,0.31,0.7,0.8)$ \\
\hline 63 & VIPERS PDR2 & 0.86 & $0.46 \pm 0.09$ & {$[170]$} & 6 June 2018 & \\
\hline 64 & BOSS DR12 voids & 0.57 & $0.501 \pm 0.051$ & {$[171]$} & 1 April 2019 & $\left(\Omega_{b}, \Omega_{m}, h, \sigma_{8}\right)=(0.0482,0.307,0.6777,0.8228)$ \\
\hline 65 & 2MTF 6dFGSv & 0.03 & $0.404 \pm 0.0815$ & [172] & 7 June 2019 & $\left(\Omega_{b}, \Omega_{m}, h, \sigma_{8}\right)=(0.0491,0.3121,0.6571,0.815)$ \\
\hline 66 & SDSS-IV & 0.72 & $0.454 \pm 0.139$ & {$[173]$} & 17 September 2019 & $\left(\Omega_{m}, \Omega_{b} h^{2}, \sigma_{8}\right)=(0.31,0.022,0.8)$ \\
\hline
\end{tabular}

TABLE VII: The $E_{G}(z)$ data compilation used in the present analysis.

The subset of the datapoints with less correlation is indicated with bold

font in the index.

\begin{tabular}{|c|c|c|c|c|c|c|}
\hline Index & Dataset & $z$ & $E_{G}(z)$ & $\sigma_{E_{G}}$ & Scale $[\mathrm{Mpc} / \mathrm{h}]$ & Reference \\
\hline \hline $\mathbf{1}$ & KiDS GAMA & 0.267 & 0.43 & 0.13 & $5<R<40$ & {$[174]$} \\
2 & SDSS BOSS LOWZ & 0.27 & 0.40 & 0.05 & $25<R<150$ & {$[175]$} \\
3 & CMB lens BOSS LOWZ & 0.27 & 0.46 & 0.085 & $25<R<150$ & {$[175]$} \\
$\mathbf{4}$ & KiDS 2dFLenS BOSS LOWZ 2dFLOZ & 0.305 & 0.27 & 0.08 & $5<R<60$ & {$[174]$} \\
$\mathbf{5}$ & RCSLenS CFHTLenS WiggleZ BOSS WGZLoZ LOWZ & 0.32 & 0.40 & 0.09 & $R>3$ & {$[176]$} \\
6 & RCSLenS CFHTLenS WiggleZ BOSS WGZLoZ LOWZ & 0.32 & 0.48 & 0.10 & $R>10$ & {$[176]$} \\
7 & SDSS & 0.32 & 0.39 & 0.06 & $10<R p<50$ & {$[70]$} \\
$\mathbf{8}$ & KiDS 2dFLenS BOSS CMASS 2dFHIZ & 0.554 & 0.26 & 0.07 & $5<R<60$ & {$[174]$} \\
$\mathbf{9}$ & RCSLenS CFHTLenS WiggleZ BOSS WGZHiZ CMASS & 0.57 & 0.31 & 0.06 & $R>3$ & {$[176]$} \\
$\mathbf{1 0}$ & RCSLenS CFHTLenS WiggleZ BOSS WGZHiZ CMASS & 0.57 & 0.30 & 0.07 & $R>10$ & {$[176]$} \\
11 & SDSS-III BOSS CMB lens CMASS & 0.57 & 0.24 & 0.06 & $R>150$ & {$[73]$} \\
12 & CFHTLenS SDSS-III BOSS CMASS & 0.57 & 0.42 & 0.056 & $5<R<26$ & {$[177]$} \\
13 & CMB lens BOSS CMASS & 0.57 & 0.39 & 0.05 & $25<R<150$ & {$[175]$} \\
14 & CFHTLenS BOSS CMASS & 0.57 & 0.43 & 0.10 & $10<R<60$ & {$[178]$}
\end{tabular}




\begin{tabular}{|l|l|l|l|l|l|l|}
$\mathbf{1 5}$ & CFHTLenS VIPERS & 0.60 & 0.16 & 0.09 & $3<R<20$ & {$[68]$} \\
$\mathbf{1 6}$ & CFHTLenS VIPERS & 0.86 & 0.09 & 0.07 & $3<R<20$ & {$[68]$} \\
\hline
\end{tabular}

TABLE VIII: The $E_{G}(R)$ data compilation in the range $0.15<z<0.43$ used in the present analysis.

\begin{tabular}{|c|c|c|c|c|c|}
\hline Index & $R[M p c / h]$ & $E_{G}(R)$ & $\sigma_{E_{G}}$ & $\mathrm{z}$ & Reference \\
\hline \hline 1 & 3.61 & 0.37 & 0.10 & 0.27 & {$[175]$} \\
2 & 4.91 & 0.42 & 0.08 & 0.27 & {$[175]$} \\
3 & 6.60 & 0.50 & 0.07 & 0.27 & {$[175]$} \\
4 & 9.07 & 0.39 & 0.07 & 0.27 & {$[175]$} \\
5 & 12.20 & 0.37 & 0.06 & 0.27 & {$[175]$} \\
6 & 16.58 & 0.45 & 0.06 & 0.27 & {$[175]$} \\
7 & 22.54 & 0.32 & 0.04 & 0.27 & {$[175]$} \\
8 & 30.30 & 0.39 & 0.05 & 0.27 & {$[175]$} \\
9 & 41.19 & 0.44 & 0.06 & 0.27 & {$[175]$} \\
10 & 55.99 & 0.45 & 0.08 & 0.27 & {$[175]$} \\
11 & 76.98 & 0.34 & 0.10 & 0.27 & {$[175]$} \\
12 & 103.47 & 0.28 & 0.15 & 0.27 & {$[175]$} \\
13 & 2.45 & 0.28 & 0.23 & 0.32 & {$[70]$} \\
14 & 3.41 & 0.49 & 0.16 & 0.32 & {$[70]$} \\
15 & 4.64 & 0.50 & 0.12 & 0.32 & {$[70]$} \\
16 & 6.62 & 0.32 & 0.09 & 0.32 & {$[70]$} \\
17 & 9.85 & 0.34 & 0.07 & 0.32 & {$[70]$} \\
18 & 14.83 & 0.45 & 0.08 & 0.32 & {$[70]$} \\
19 & 22.10 & 0.43 & 0.09 & 0.32 & {$[70]$} \\
20 & 45.87 & 0.32 & 0.10 & 0.32 & {$[70]$} \\
21 & 1.76 & 0.74 & 0.21 & $0.15-0.43$ & {$[176]$} \\
22 & 2.23 & 0.71 & 0.15 & $0.15-0.43$ & {$[176]$} \\
23 & 2.85 & 0.35 & 0.14 & $0.15-0.43$ & {$[176]$} \\
24 & 3.56 & 0.30 & 0.11 & $0.15-0.43$ & {$[176]$} \\
25 & 4.45 & 0.35 & 0.11 & $0.15-0.43$ & {$[176]$} \\
26 & 5.65 & 0.28 & 0.10 & $0.15-0.43$ & {$[176]$} \\
27 & 7.059 & 0.43 & 0.11 & $0.15-0.43$ & {$[176]$} \\
28 & 8.94 & 0.45 & 0.11 & $0.15-0.43$ & {$[176]$} \\
29 & 11.33 & 0.47 & 0.12 & $0.15-0.43$ & {$[176]$} \\
30 & 14.34 & 0.55 & 0.12 & $0.15-0.43$ & {$[176]$} \\
31 & 17.98 & 0.40 & 0.12 & $0.15-0.43$ & {$[176]$} \\
32 & 22.21 & 0.37 & 0.14 & $0.15-0.43$ & {$[176]$} \\
33 & 28.88 & 0.39 & 0.18 & $0.15-0.43$ & {$[176]$} \\
34 & 36.15 & 0.35 & 0.19 & $0.15-0.43$ & {$[176]$} \\
35 & 45.26 & 0.30 & 0.30 & $0.15-0.43$ & {$[176]$} \\
36 & 5.01 & 0.25 & 0.16 & $0.15-0.43$ & {$[174]$} \\
37 & 5.37 & 0.39 & 0.16 & $0.15-0.43$ & {$[174]$} \\
38 & 5.58 & 0.094 & 0.18 & $0.15-0.43$ & {$[174]$} \\
39 & 8.15 & 0.30 & 0.14 & $0.15-0.43$ & {$[174]$} \\
40 & 8.57 & 0.41 & 0.14 & $0.15-0.43$ & {$[174]$} \\
41 & 9.02 & 0.41 & 0.24 & $0.15-0.43$ & {$[174]$} \\
42 & 13.23 & 0.49 & 0.16 & $0.15-0.43$ & {$[174]$} \\
43 & 13.95 & 0.43 & 0.16 & $0.15-0.43$ & {$[174]$} \\
44 & 14.76 & 0.15 & 0.17 & $0.15-0.43$ & {$[174]$} \\
45 & 21.08 & 0.51 & 0.23 & $0.15-0.43$ & {$[174]$} \\
46 & 22.75 & 0.33 & 0.23 & $0.15-0.43$ & {$[174]$} \\
47 & 23.96 & 0.32 & 0.32 & $0.15-0.43$ & {$[174]$} \\
48 & 35.52 & 0.33 & 0.29 & $0.15-0.43$ & {$[174]$} \\
49 & 36.98 & 0.40 & 0.33 & $0.15-0.43$ & {$[174]$} \\
50 & 39.00 & 0.32 & 0.38 & $0.15-0.43$ & {$[174]$} \\
51 & 56.60 & 0.37 & 0.80 & $0.15-0.43$ & {$[174]$} \\
\hline & & & & & \\
\hline
\end{tabular}

TABLE IX: The $E_{G}(R)$ data compilation in the range $0.43<z<1.2$ used in the present analysis.

$$
\begin{array}{|l|l|l|l|l|l|}
\hline \text { Index } & R[M p c / h] & E_{G}(R) & \sigma_{E_{G}} & \mathrm{z} & \text { Reference } \\
\hline
\end{array}
$$




\begin{tabular}{|c|c|c|c|c|c|}
\hline 1 & 5.13 & 0.23 & \begin{tabular}{|l|}
0.14 \\
\end{tabular} & $0.43-0.7$ & [174] \\
\hline 2 & 5.69 & 0.19 & 0.19 & $0.43-0.7$ & [174] \\
\hline 3 & 8.28 & 0.32 & 0.12 & $0.43-0.7$ & [174] \\
\hline 4 & 9.19 & 0.27 & 0.17 & $0.43-0.7$ & [174] \\
\hline 5 & 13.69 & 0.21 & 0.12 & $0.43-0.7$ & [174] \\
\hline 6 & 14.98 & 0.46 & 0.25 & $0.43-0.7$ & [174] \\
\hline 7 & 24.43 & 0.95 & 0.47 & $0.43-0.7$ & [174] \\
\hline 8 & 22.02 & 0.22 & 0.13 & $0.43-0.7$ & {$[174]$} \\
\hline 9 & 36.28 & 0.48 & 0.18 & $0.43-0.7$ & [174] \\
\hline 10 & 39.84 & 0.84 & 0.57 & $0.43-0.7$ & [174] \\
\hline 11 & 59.78 & 0.54 & 0.45 & $0.43-0.7$ & [174] \\
\hline 12 & 1.74 & 0.34 & 0.29 & $0.43-0.7$ & {$[176]$} \\
\hline 13 & 2.25 & 0.31 & 0.17 & $0.43-0.7$ & {$[176]$} \\
\hline 14 & 2.74 & 0.57 & 0.14 & $0.43-0.7$ & [176] \\
\hline 15 & 3.46 & 0.43 & 0.11 & $0.43-0.7$ & [176] \\
\hline 16 & 4.45 & 0.35 & 0.10 & $0.43-0.7$ & [176] \\
\hline 17 & 5.56 & 0.30 & 0.09 & $0.43-0.7$ & {$[176]$} \\
\hline 18 & 6.92 & 0.24 & 0.09 & $0.43-0.7$ & [176] \\
\hline 19 & 8.84 & 0.28 & 0.08 & $0.43-0.7$ & [176] \\
\hline 20 & 11.15 & 0.26 & 0.08 & $0.43-0.7$ & [176] \\
\hline 21 & 13.90 & 0.29 & 0.07 & $0.43-0.7$ & [176] \\
\hline 22 & 17.74 & 0.24 & 0.08 & $0.43-0.7$ & [176] \\
\hline 23 & 21.96 & 0.25 & 0.09 & $0.43-0.7$ & {$[176]$} \\
\hline 24 & 27.80 & 0.32 & 0.10 & $0.43-0.7$ & [176] \\
\hline 25 & 34.81 & 0.41 & 0.14 & $0.43-0.7$ & [176] \\
\hline 26 & 44.26 & 0.62 & 0.19 & $0.43-0.7$ & {$[176]$} \\
\hline 27 & 2.17 & 0.38 & 0.15 & 0.57 & [177] \\
\hline 28 & 3.30 & 0.26 & 0.11 & 0.57 & [177] \\
\hline 29 & 4.99 & 0.46 & 0.09 & 0.57 & [177] \\
\hline 30 & 7.56 & 0.42 & 0.08 & 0.57 & [177] \\
\hline 31 & 11.38 & 0.35 & 0.08 & 0.57 & [177] \\
\hline 32 & 17.26 & 0.43 & 0.09 & 0.57 & [177] \\
\hline 33 & 25.99 & 0.43 & 0.11 & 0.57 & [177] \\
\hline 34 & 39.60 & 0.33 & 0.15 & 0.57 & [177] \\
\hline 35 & 2.56 & 0.48 & 0.08 & 0.57 & [175] \\
\hline 36 & 3.40 & 0.36 & 0.09 & 0.57 & [175] \\
\hline 37 & 4.60 & 0.32 & 0.08 & 0.57 & [175] \\
\hline 38 & 6.12 & 0.40 & 0.08 & 0.57 & [175] \\
\hline 39 & 8.20 & 0.33 & 0.07 & 0.57 & [175] \\
\hline 40 & 11.00 & 0.43 & 0.09 & 0.57 & [175] \\
\hline 41 & 14.87 & 0.32 & 0.07 & 0.57 & [175] \\
\hline 42 & 19.75 & 0.34 & 0.12 & 0.57 & [175] \\
\hline 43 & 26.21 & 0.31 & 0.15 & 0.57 & [175] \\
\hline 44 & 35.44 & 0.22 & 0.18 & 0.57 & [175] \\
\hline 45 & 2.22 & 0.27 & 0.17 & 0.57 & [178] \\
\hline 46 & 3.56 & 0.13 & 0.23 & 0.57 & [178] \\
\hline 47 & 5.64 & 0.16 & 0.19 & 0.57 & [178] \\
\hline 48 & 8.86 & 0.49 & 0.28 & 0.57 & [178] \\
\hline 49 & 14.13 & 0.64 & 0.22 & 0.57 & [178] \\
\hline 50 & 22.54 & 0.29 & 0.14 & 0.57 & [178] \\
\hline 51 & 35.37 & 0.40 & 0.21 & 0.57 & [178] \\
\hline 52 & 2.64 & 0.31 & 0.14 & $0.5-0.7$ & {$[68]$} \\
\hline 53 & 4.17 & 0.20 & 0.15 & $0.5-0.7$ & [68] \\
\hline 54 & 6.63 & 0.22 & 0.17 & $0.5-0.7$ & [68] \\
\hline 55 & 10.44 & 0.01 & 0.20 & $0.5-0.7$ & [68] \\
\hline 56 & 16.37 & 0.09 & 0.26 & $0.5-0.7$ & [68] \\
\hline 57 & 2.61 & 0.34 & 0.12 & $0.7-1.2$ & [68] \\
\hline 58 & 4.15 & 0.06 & 0.12 & $0.7-1.2$ & [68] \\
\hline 59 & 6.60 & 0.11 & 0.13 & $0.7-1.2$ & [68] \\
\hline 60 & 10.42 & 0.01 & 0.16 & $0.7-1.2$ & [68] \\
\hline 61 & 16.56 & 0.10 & 0.21 & $0.7-1.2$ & [68] \\
\hline
\end{tabular}


[1] Savvas Nesseris, George Pantazis, and Leandros Perivolaropoulos, "Tension and constraints on modified gravity parametrizations of $G_{\text {eff }}(z)$ from growth rate and Planck data," Phys. Rev. D96, 023542 (2017), arXiv:1703.10538 [astro-ph.CO].

[2] Lavrentios Kazantzidis and Leandros Perivolaropoulos, "Evolution of the $f \sigma_{8}$ tension with the Planck15/ $\Lambda \mathrm{CDM}$ determination and implications for modified gravity theories," Phys. Rev. D97, 103503 (2018), arXiv:1803.01337 [astro-ph.CO].

[3] Leandros Perivolaropoulos and Lavrentios Kazantzidis, "Hints of modified gravity in cosmos and in the lab?" Int. J. Mod. Phys. D28, 1942001 (2019), arXiv:1904.09462 [gr-qc].

[4] Lavrentios Kazantzidis and Leandros Perivolaropoulos, "Is gravity getting weaker at low $z$ ? Observational evidence and theoretical implications," (2019), arXiv:1907.03176 [astro-ph.CO].

[5] Sean M. Carroll, "The Cosmological constant," Living Rev. Rel. 4, 1 (2001), arXiv:astro-ph/0004075 [astro$\mathrm{ph}$.

[6] Clifford M. Will, "The Confrontation between General Relativity and Experiment," Living Rev. Rel. 17, 4 (2014), arXiv:1403.7377 [gr-qc].

[7] Adam G. Riess et al. (Supernova Search Team), "Observational evidence from supernovae for an accelerating universe and a cosmological constant," Astron. J. 116, 1009-1038 (1998), arXiv:astro-ph/9805201 [astro-ph].

[8] S. Perlmutter et al. (Supernova Cosmology Project), "Measurements of $\Omega$ and $\Lambda$ from 42 high redshift supernovae," Astrophys. J. 517, 565-586 (1999), arXiv:astroph/9812133 [astro-ph].

[9] Steven Weinberg, "The Cosmological Constant Problem," Rev. Mod. Phys. 61, 1-23 (1989).

[10] Jerome Martin, "Everything You Always Wanted To Know About The Cosmological Constant Problem (But Were Afraid To Ask)," Comptes Rendus Physique 13, 566-665 (2012), arXiv:1205.3365 [astro-ph.CO].

[11] C. P. Burgess, "The Cosmological Constant Problem: Why it's hard to get Dark Energy from Micro-physics," in Proceedings, 100th Les Houches Summer School: Post-Planck Cosmology: Les Houches, France, July 8 - August 2, 2013 (2015) pp. 149-197, arXiv:1309.4133 [hep-th].

[12] P. J. Steinhardt, "Cosmological Challenges for the 21st Century," in Critical Problems in Physics, edited by V. L. Fitch, D. R. Marlow, and M. A. E. Dementi (1997) p. 123.

[13] H. E. S. Velten, R. F. vom Marttens, and W. Zimdahl, "Aspects of the cosmological "coincidence problem"," Eur. Phys. J. C74, 3160 (2014), arXiv:1410.2509 [astroph.CO].

[14] P. A. R. Ade et al. (Planck), "Planck 2015 results. XIII. Cosmological parameters," Astron. Astrophys. 594, A13 (2016), arXiv:1502.01589 [astro-ph.CO].

[15] N. Aghanim et al. (Planck), "Planck 2018 results. VI. Cosmological parameters," (2018), arXiv:1807.06209 [astro-ph.CO].

[16] Adam G. Riess et al., "A 2.4\% Determination of the Local Value of the Hubble Constant," Astrophys. J. 826, 56 (2016), arXiv:1604.01424 [astro-ph.CO].
[17] Adam G. Riess et al., "Milky Way Cepheid Standards for Measuring Cosmic Distances and Application to Gaia DR2: Implications for the Hubble Constant," Astrophys. J. 861, 126 (2018), arXiv:1804.10655 [astroph.CO].

[18] S. Birrer et al., "H0LiCOW - IX. Cosmographic analysis of the doubly imaged quasar SDSS $1206+4332$ and a new measurement of the Hubble constant," Mon. Not. Roy. Astron. Soc. 484, 4726 (2019), arXiv:1809.01274 [astro-ph.CO].

[19] Gong-Bo Zhao, Levon Pogosian, Alessandra Silvestri, and Joel Zylberberg, "Searching for modified growth patterns with tomographic surveys," Phys. Rev. D79, 083513 (2009), arXiv:0809.3791 [astro-ph].

[20] Yan-Chuan Cai and Gary Bernstein, "Combining weak lensing tomography and spectroscopic redshift surveys," Mon. Not. Roy. Astron. Soc. 422, 1045-1056 (2012), arXiv:1112.4478 [astro-ph.CO].

[21] Shahab Joudaki and Manoj Kaplinghat, "Dark Energy and Neutrino Masses from Future Measurements of the Expansion History and Growth of Structure," Phys. Rev. D86, 023526 (2012), arXiv:1106.0299 [astroph.CO].

[22] Ismael Tereno, Elisabetta Semboloni, and Tim Schrabback, "COSMOS weak-lensing constraints on modified gravity," Astron. Astrophys. 530, A68 (2011), arXiv:1012.5854 [astro-ph.CO].

[23] Fergus Simpson et al., "CFHTLenS: Testing the Laws of Gravity with Tomographic Weak Lensing and Redshift Space Distortions," Mon. Not. Roy. Astron. Soc. 429, 2249 (2013), arXiv:1212.3339 [astro-ph.CO].

[24] Gongbo Zhao, David Bacon, Roy Maartens, Mario Santos, and Alvise Raccanelli, "Model-independent constraints on dark energy and modified gravity with the SKA," Proceedings, Advancing Astrophysics with the Square Kilometre Array (AASKA14): Giardini Naxos, Italy, June 9-13, 2014, PoS AASKA14, 165 (2015).

[25] Shahab Joudaki et al., "KiDS-450 + 2dFLenS: Cosmological parameter constraints from weak gravitational lensing tomography and overlapping redshiftspace galaxy clustering," Mon. Not. Roy. Astron. Soc. 474, 4894-4924 (2018), arXiv:1707.06627 [astroph.CO].

[26] Edmund Bertschinger, "On the Growth of Perturbations as a Test of Dark Energy," Astrophys. J. 648, 797-806 (2006), arXiv:astro-ph/0604485 [astro-ph].

[27] S. Nesseris and Leandros Perivolaropoulos, "Crossing the Phantom Divide: Theoretical Implications and Observational Status," JCAP 0701, 018 (2007), arXiv:astro-ph/0610092 [astro-ph].

[28] Spyros Basilakos, Savvas Nesseris, and Leandros Perivolaropoulos, "Observational constraints on viable $\mathrm{f}(\mathrm{R})$ parametrizations with geometrical and dynamical probes," Phys. Rev. D87, 123529 (2013), arXiv:1302.6051 [astro-ph.CO].

[29] Eduardo J. Ruiz and Dragan Huterer, "Testing the dark energy consistency with geometry and growth," Phys. Rev. D91, 063009 (2015), arXiv:1410.5832 [astroph.CO].

[30] Eduardo Rozo et al. (DSDD), "Cosmological Constraints from the SDSS maxBCG Cluster Catalog," As- 
trophys. J. 708, 645-660 (2010), arXiv:0902.3702 [astroph.CO].

[31] David Rapetti, Steven W. Allen, Adam Mantz, and Harald Ebeling, "Constraints on modified gravity from the observed X-ray luminosity function of galaxy clusters," Mon. Not. Roy. Astron. Soc. 400, 699 (2009), arXiv:0812.2259 [astro-ph].

[32] S. Bocquet et al. (SPT), "Mass Calibration and Cosmological Analysis of the SPT-SZ Galaxy Cluster Sample Using Velocity Dispersion $\sigma_{v}$ and X-ray $Y_{\mathrm{X}}$ Measurements," Astrophys. J. 799, 214 (2015), arXiv:1407.2942 [astro-ph.CO].

[33] Fabian Schmidt, "Weak Lensing Probes of Modified Gravity," Phys. Rev. D78, 043002 (2008), arXiv:0805.4812 [astro-ph].

[34] H. Hildebrandt et al., "KiDS-450: Cosmological parameter constraints from tomographic weak gravitational lensing," Mon. Not. Roy. Astron. Soc. 465, 1454 (2017), arXiv:1606.05338 [astro-ph.CO].

[35] Catherine Heymans et al., "CFHTLenS: The CanadaFrance-Hawaii Telescope Lensing Survey," Mon. Not. Roy. Astron. Soc. 427, 146 (2012), arXiv:1210.0032 [astro-ph.CO].

[36] M. A. Troxel et al. (DES), "Dark Energy Survey Year 1 results: Cosmological constraints from cosmic shear," Phys. Rev. D98, 043528 (2018), arXiv:1708.01538 [astro-ph.CO].

[37] F. Köhlinger et al., "KiDS-450: The tomographic weak lensing power spectrum and constraints on cosmological parameters," Mon. Not. Roy. Astron. Soc. 471, 44124435 (2017), arXiv:1706.02892 [astro-ph.CO].

[38] T. M. C. Abbott et al. (DES), "Dark Energy Survey year 1 results: Cosmological constraints from galaxy clustering and weak lensing," Phys. Rev. D98, 043526 (2018), arXiv:1708.01530 [astro-ph.CO].

[39] T. M. C. Abbott et al. (DES), "Dark Energy Survey Year 1 Results: Constraints on Extended Cosmological Models from Galaxy Clustering and Weak Lensing," Phys. Rev. D99, 123505 (2019), arXiv:1810.02499 [astro-ph.CO].

[40] Lado Samushia et al., "The Clustering of Galaxies in the SDSS-III DR9 Baryon Oscillation Spectroscopic Survey: Testing Deviations from $\Lambda$ and General Relativity using anisotropic clustering of galaxies," Mon. Not. Roy. Astron. Soc. 429, 1514-1528 (2013), arXiv:1206.5309 [astro-ph.CO].

[41] Edward Macaulay, Ingunn Kathrine Wehus, and Hans Kristian Eriksen, "Lower Growth Rate from Recent Redshift Space Distortion Measurements than Expected from Planck," Phys. Rev. Lett. 111, 161301 (2013), arXiv:1303.6583 [astro-ph.CO].

[42] Andrew Johnson, Chris Blake, Jason Dossett, Jun Koda, David Parkinson, and Shahab Joudaki, "Searching for Modified Gravity: Scale and Redshift Dependent Constraints from Galaxy Peculiar Velocities," Mon. Not. Roy. Astron. Soc. 458, 2725-2744 (2016), arXiv:1504.06885 [astro-ph.CO].

[43] Suresh Kumar and Rafael C. Nunes, "Probing the interaction between dark matter and dark energy in the presence of massive neutrinos," Phys. Rev. D94, 123511 (2016), arXiv:1608.02454 [astro-ph.CO].

[44] Alkistis Pourtsidou and Thomas Tram, "Reconciling $\mathrm{CMB}$ and structure growth measurements with dark energy interactions," Phys. Rev. D94, 043518 (2016),
arXiv:1604.04222 [astro-ph.CO].

[45] Bruno J. Barros, Luca Amendola, Tiago Barreiro, and Nelson J. Nunes, "Coupled quintessence with a $\Lambda$ CDM background: removing the $\sigma_{8}$ tension," JCAP 1901, 007 (2019), arXiv:1802.09216 [astro-ph.CO].

[46] Stefano Camera, Matteo Martinelli, and Daniele Bertacca, "Does quartessence ease cosmic tensions?" Phys. Dark Univ. 23, 100247 (2019), arXiv:1704.06277 [astro-ph.CO].

[47] Weiqiang Yang, Supriya Pan, Eleonora Di Valentino, Emmanuel N. Saridakis, and Subenoy Chakraborty, "Observational constraints on one-parameter dynamical dark-energy parametrizations and the $H_{0}$ tension," Phys. Rev. D99, 043543 (2019), arXiv:1810.05141 [astro-ph.CO].

[48] Gaetano Lambiase, Subhendra Mohanty, Ashish Narang, and Priyank Parashari, "Testing dark energy models in the light of $\sigma_{8}$ tension," Eur. Phys. J. C79, 141 (2019), arXiv:1804.07154 [astro-ph.CO].

[49] Adria Gomez-Valent and Joan Sola, "Relaxing the $\sigma_{8}$ tension through running vacuum in the Universe," EPL 120, 39001 (2017), arXiv:1711.00692 [astro-ph.CO].

[50] Adrià Gómez-Valent and Joan Solà, "Density perturbations for running vacuum: a successful approach to structure formation and to the $\sigma_{8}$-tension," (2018), arXiv:1801.08501 [astro-ph.CO].

[51] Ana Diaz Rivero, V. Miranda, and Cora Dvorkin, "Observable Predictions for Massive-Neutrino Cosmologies with Model-Independent Dark Energy," Phys. Rev. D100, 063504 (2019), arXiv:1903.03125 [astro-ph.CO].

[52] Shinji Tsujikawa, "Matter density perturbations and effective gravitational constant in modified gravity models of dark energy," Phys. Rev. D76, 023514 (2007), arXiv:0705.1032 [astro-ph].

[53] Wayne $\mathrm{Hu}$ and Ignacy Sawicki, "Models of $\mathrm{f}(\mathrm{R})$ Cosmic Acceleration that Evade Solar-System Tests," Phys. Rev. D76, 064004 (2007), arXiv:0705.1158 [astro-ph].

[54] R. Gannouji, B. Moraes, and D. Polarski, "The growth of matter perturbations in $\mathrm{f}(\mathrm{R})$ models," JCAP 0902, 034 (2009), arXiv:0809.3374 [astro-ph].

[55] Salvatore Capozziello and Mariafelicia De Laurentis, "Extended Theories of Gravity," Phys. Rept. 509, 167321 (2011), arXiv:1108.6266 [gr-qc].

[56] Timothy Clifton, Pedro G. Ferreira, Antonio Padilla, and Constantinos Skordis, "Modified Gravity and Cosmology," Phys. Rept. 513, 1-189 (2012), arXiv:1106.2476 [astro-ph.CO].

[57] Shin'ichi Nojiri and Sergei D. Odintsov, "Unified cosmic history in modified gravity: from $\mathrm{F}(\mathrm{R})$ theory to Lorentz non-invariant models," Phys. Rept. 505, 59144 (2011), arXiv:1011.0544 [gr-qc].

[58] Lotfi Boubekeur, Elena Giusarma, Olga Mena, and Héctor Ramírez, "Current status of modified gravity," Phys. Rev. D90, 103512 (2014), arXiv:1407.6837 [astroph.CO].

[59] Yi-Fu Cai, Chunlong Li, Emmanuel N. Saridakis, and Lingqin Xue, " $f(T)$ gravity after GW170817 and GRB170817A," Phys. Rev. D97, 103513 (2018), arXiv:1801.05827 [gr-qc].

[60] Judit Pérez-Romero and Savvas Nesseris, "Cosmological constraints and comparison of viable $f(R)$ models," Phys. Rev. D97, 023525 (2018), arXiv:1710.05634 [astro-ph.CO].

[61] S. Nojiri, S. D. Odintsov, and V. K. Oikonomou, 
"Modified Gravity Theories on a Nutshell: Inflation, Bounce and Late-time Evolution," Phys. Rept. 692, 1104 (2017), arXiv:1705.11098 [gr-qc].

[62] Yong-Seon Song, Wayne Hu, and Ignacy Sawicki, "The Large Scale Structure of $\mathrm{f}(\mathrm{R})$ Gravity," Phys. Rev. D75, 044004 (2007), arXiv:astro-ph/0610532 [astro-ph].

[63] Philippe Brax, Carsten van de Bruck, Anne-Christine Davis, and Douglas J. Shaw, "f(R) Gravity and Chameleon Theories," Phys. Rev. D78, 104021 (2008), arXiv:0806.3415 [astro-ph].

[64] Kazuya Koyama, "Cosmological Tests of Modified Gravity," Rept. Prog. Phys. 79, 046902 (2016), arXiv:1504.04623 [astro-ph.CO].

[65] Mustapha Ishak, "Testing General Relativity in Cosmology," Living Rev. Rel. 22, 1 (2019), arXiv:1806.10122 [astro-ph.CO].

[66] Pengjie Zhang, Michele Liguori, Rachel Bean, and Scott Dodelson, "Probing Gravity at Cosmological Scales by Measurements which Test the Relationship between Gravitational Lensing and Matter Overdensity," Phys. Rev. Lett. 99, 141302 (2007), arXiv:0704.1932 [astro$\mathrm{ph}]$.

[67] C. Danielle Leonard, Pedro G. Ferreira, and Catherine Heymans, "Testing gravity with $E_{G}$ : mapping theory onto observations," JCAP 1512, 051 (2015), arXiv:1510.04287 [astro-ph.CO].

[68] S. de la Torre et al., "The VIMOS Public Extragalactic Redshift Survey (VIPERS). Gravity test from the combination of redshift-space distortions and galaxy-galaxy lensing at $0.5<z<1.2$," Astron. Astrophys. 608, A44 (2017), arXiv:1612.05647 [astro-ph.CO].

[69] James M. Bardeen, "Gauge Invariant Cosmological Perturbations," Phys. Rev. D22, 1882-1905 (1980).

[70] Reinabelle Reyes, Rachel Mandelbaum, Uros Seljak, Tobias Baldauf, James E. Gunn, Lucas Lombriser, and Robert E. Smith, "Confirmation of general relativity on large scales from weak lensing and galaxy velocities," Nature 464, 256-258 (2010), arXiv:1003.2185 [astroph.CO].

[71] P. A. R. Ade et al. (Planck), "Planck 2015 results. XV. Gravitational lensing," Astron. Astrophys. 594, A15 (2016), arXiv:1502.01591 [astro-ph.CO].

[72] Anthony R. Pullen, Shadab Alam, and Shirley Ho, "Probing gravity at large scales through CMB lensing," Mon. Not. Roy. Astron. Soc. 449, 4326-4335 (2015), arXiv:1412.4454 [astro-ph.CO].

[73] Anthony R. Pullen, Shadab Alam, Siyu He, and Shirley Ho, "Constraining Gravity at the Largest Scales through CMB Lensing and Galaxy Velocities," Mon. Not. Roy. Astron. Soc. 460, 4098-4108 (2016), arXiv:1511.04457 [astro-ph.CO].

[74] Matthias Bartelmann and Peter Schneider, "Weak gravitational lensing," Phys. Rept. 340, 291-472 (2001), arXiv:astro-ph/9912508 [astro-ph].

[75] Henk Hoekstra, Howard K. C. Yee, and Michael D. Gladders, "Properties of galaxy dark matter halos from weak lensing," Astrophys. J. 606, 67-77 (2004), arXiv:astro-ph/0306515 [astro-ph].

[76] Rachel Mandelbaum, Christopher M. Hirata, Uros Seljak, Jacek Guzik, Nikhil Padmanabhan, Cullen Blake, Michael R. Blanton, Robert Lupton, and Jonathan Brinkmann, "Systematic errors in weak lensing: Application to SDSS galaxy-galaxy weak lensing," Mon. Not. Roy. Astron. Soc. 361, 1287-1322 (2005), arXiv:astro- ph/0501201 [astro-ph].

[77] Martin Kilbinger, "Cosmology with cosmic shear observations: a review," Rept. Prog. Phys. 78, 086901 (2015), arXiv:1411.0115 [astro-ph.CO].

[78] Viatcheslav F. Mukhanov, H. A. Feldman, and Robert H. Brandenberger, "Theory of cosmological perturbations. Part 1. Classical perturbations. Part 2. Quantum theory of perturbations. Part 3. Extensions," Phys. Rept. 215, 203-333 (1992).

[79] Chung-Pei Ma and Edmund Bertschinger, "Cosmological perturbation theory in the synchronous and conformal Newtonian gauges," Astrophys. J. 455, 7-25 (1995), arXiv:astro-ph/9506072 [astro-ph].

[80] Gilles Esposito-Farese and D. Polarski, "Scalar tensor gravity in an accelerating universe," Phys. Rev. D63, 063504 (2001), arXiv:gr-qc/0009034 [gr-qc].

[81] N. Kaiser, "Clustering in real space and in redshift space," Mon. Not. Roy. Astron. Soc. 227, 1-27 (1987).

[82] A. J. S. Hamilton, "Linear redshift distortions: A Review," in Ringberg Workshop on Large Scale Structure Ringberg, Germany, September 23-28, 1996 (1997) arXiv:astro-ph/9708102 [astro-ph].

[83] Lado Samushia et al., "The clustering of galaxies in the SDSS-III Baryon Oscillation Spectroscopic Survey: measuring growth rate and geometry with anisotropic clustering," Mon. Not. Roy. Astron. Soc. 439, 35043519 (2014), arXiv:1312.4899 [astro-ph.CO].

[84] Florian Beutler, Chris Blake, Matthew Colless, D. Heath Jones, Lister Staveley-Smith, Gregory B. Poole, Lachlan Campbell, Quentin Parker, Will Saunders, and Fred Watson, "The 6dF Galaxy Survey: $z \approx 0$ measurement of the growth rate and $\sigma_{8}$," Mon. Not. Roy. Astron. Soc. 423, 3430-3444 (2012), arXiv:1204.4725 [astro-ph.CO].

[85] Edmund Bertschinger, "One Gravitational Potential or Two? Forecasts and Tests," Phil. Trans. Roy. Soc. Lond. A369, 4947-4961 (2011), arXiv:1111.4659 [astroph.CO].

[86] Edmund Bertschinger and Phillip Zukin, "Distinguishing Modified Gravity from Dark Energy," Phys. Rev. D78, 024015 (2008), arXiv:0801.2431 [astro-ph].

[87] Gong-Bo Zhao, Tommaso Giannantonio, Levon Pogosian, Alessandra Silvestri, David J. Bacon, Kazuya Koyama, Robert C. Nichol, and Yong-Seon Song, "Probing modifications of General Relativity using current cosmological observations," Phys. Rev. D81, 103510 (2010), arXiv:1003.0001 [astro-ph.CO].

[88] Scott F. Daniel, Eric V. Linder, Tristan L. Smith, Robert R. Caldwell, Asantha Cooray, Alexie Leauthaud, and Lucas Lombriser, "Testing General Relativity with Current Cosmological Data," Phys. Rev. D81, 123508 (2010), arXiv:1002.1962 [astro-ph.CO].

[89] Yong-Seon Song, Gong-Bo Zhao, David Bacon, Kazuya Koyama, Robert C. Nichol, and Levon Pogosian, "Complementarity of Weak Lensing and Peculiar Velocity Measurements in Testing General Relativity," Phys. Rev. D84, 083523 (2011), arXiv:1011.2106 [astroph.CO].

[90] Scott F. Daniel and Eric V. Linder, "Confronting General Relativity with Further Cosmological Data," Phys. Rev. D82, 103523 (2010), arXiv:1008.0397 [astroph.CO].

[91] Bin Hu, Michele Liguori, Nicola Bartolo, and Sabino Matarrese, "Parametrized modified gravity con- 
straints after Planck," Phys. Rev. D88, 123514 (2013), arXiv:1307.5276 [astro-ph.CO].

[92] Agnès Ferté, Donnacha Kirk, Andrew R. Liddle, and Joe Zuntz, "Testing gravity on cosmological scales with cosmic shear, cosmic microwave background anisotropies, and redshift-space distortions," Phys. Rev. D99, 083512 (2019), arXiv:1712.01846 [astro-ph.CO].

[93] F. Bernardeau, S. Colombi, E. Gaztanaga, and R. Scoccimarro, "Large scale structure of the universe and cosmological perturbation theory," Phys. Rept. 367, 1-248 (2002), arXiv:astro-ph/0112551 [astro-ph].

[94] P. J. E. Peebles, Research supported by the National Science Foundation. Princeton, N.J., Princeton University Press, 1980. 435 p. (1980).

[95] Ofer Lahav, Per B. Lilje, Joel R. Primack, and Martin J. Rees, "Dynamical effects of the cosmological constant," Mon. Not. Roy. Astron. Soc. 251, 128-136 (1991).

[96] Li-Min Wang and Paul J. Steinhardt, "Cluster abundance constraints on quintessence models," Astrophys. J. 508, 483-490 (1998), arXiv:astro-ph/9804015 [astro$\mathrm{ph}$.

[97] Eric V. Linder, "Cosmic growth history and expansion history," Phys. Rev. D72, 043529 (2005), arXiv:astroph/0507263 [astro-ph].

[98] David Polarski and Radouane Gannouji, "On the growth of linear perturbations," Phys. Lett. B660, 439443 (2008), arXiv:0710.1510 [astro-ph].

[99] Eric V. Linder and Robert N. Cahn, "Parameterized Beyond-Einstein Growth," Astropart. Phys. 28, 481488 (2007), arXiv:astro-ph/0701317 [astro-ph].

[100] Radouane Gannouji and David Polarski, "The growth of matter perturbations in some scalar-tensor DE models," JCAP 0805, 018 (2008), arXiv:0802.4196 [astro-ph].

[101] Savvas Nesseris and Domenico Sapone, "Accuracy of the growth index in the presence of dark energy perturbations," Phys. Rev. D92, 023013 (2015), arXiv:1505.06601 [astro-ph.CO].

[102] David Polarski, Alexei A. Starobinsky, and Hector Giacomini, "When is the growth index constant?" JCAP 1612, 037 (2016), arXiv:1610.00363 [astro-ph.CO].

[103] Levon Pogosian and Alessandra Silvestri, "The pattern of growth in viable $\mathrm{f}(\mathrm{R})$ cosmologies," Phys. Rev. D77, 023503 (2008), [Erratum: Phys. Rev.D81,049901(2010)], arXiv:0709.0296 [astro-ph].

[104] Bhuvnesh Jain and Justin Khoury, "Cosmological Tests of Gravity," Annals Phys. 325, 1479-1516 (2010), arXiv:1004.3294 [astro-ph.CO].

[105] Luca Amendola, Martin Kunz, and Domenico Sapone, "Measuring the dark side (with weak lensing)," JCAP 0804, 013 (2008), arXiv:0704.2421 [astro-ph].

[106] Levon Pogosian, Alessandra Silvestri, Kazuya Koyama, and Gong-Bo Zhao, "How to optimally parametrize deviations from General Relativity in the evolution of cosmological perturbations?" Phys. Rev. D81, 104023 (2010), arXiv:1002.2382 [astro-ph.CO].

[107] Scott F. Daniel and Eric V. Linder, "Constraining Cosmic Expansion and Gravity with Galaxy Redshift Surveys," JCAP 1302, 007 (2013), arXiv:1212.0009 [astroph.CO].

[108] Dragan Huterer et al., "Growth of Cosmic Structure: Probing Dark Energy Beyond Expansion," Astropart. Phys. 63, 23-41 (2015), arXiv:1309.5385 [astro-ph.CO].

[109] Eva-Maria Mueller, Will Percival, Eric Linder, Shadab
Alam, Gong-Bo Zhao, Ariel G. Sánchez, Florian Beutler, and Jon Brinkmann, "The clustering of galaxies in the completed SDSS-III Baryon Oscillation Spectroscopic Survey: constraining modified gravity," Mon. Not. Roy. Astron. Soc. 475, 2122-2131 (2018), arXiv:1612.00812 [astro-ph.CO].

[110] Louis Perenon, Julien Bel, Roy Maartens, and Alvaro de la Cruz-Dombriz, "Optimising growth of structure constraints on modified gravity," JCAP 1906, 020 (2019), arXiv:1901.11063 [astro-ph.CO].

[111] Rubén Arjona, Wilmar Cardona, and Savvas Nesseris, "Unraveling the effective fluid approach for $f(R)$ models in the sub-horizon approximation," (2018), arXiv:1811.02469 [astro-ph.CO].

[112] Luca Amendola, Martin Kunz, Mariele Motta, Ippocratis D. Saltas, and Ignacy Sawicki, "Observables and unobservables in dark energy cosmologies," Phys. Rev. D87, 023501 (2013), arXiv:1210.0439 [astroph.CO].

[113] Mariele Motta, Ignacy Sawicki, Ippocratis D. Saltas, Luca Amendola, and Martin Kunz, "Probing Dark Energy through Scale Dependence," Phys. Rev. D88, 124035 (2013), arXiv:1305.0008 [astro-ph.CO].

[114] Ana Marta Pinho, Santiago Casas, and Luca Amendola, "Model-independent reconstruction of the linear anisotropic stress $\eta$," JCAP 1811, 027 (2018), arXiv:1805.00027 [astro-ph.CO].

[115] J. C. Bueno Sanchez and L. Perivolaropoulos, "Evolution of Dark Energy Perturbations in ScalarTensor Cosmologies," Phys. Rev. D81, 103505 (2010), arXiv:1002.2042 [astro-ph.CO].

[116] Jurgen Muller, James G. Williams, and Slava G. Turyshev, "Lunar laser ranging contributions to relativity and geodesy," Proceedings, 359th WE-Heraeus Seminar : Lasers, Clocks, and Drag-Free: Technologies for Future Exploration in Space and tests of Gravity: Bremen, Germany, May 30-June 1, 2005, Astrophys. Space Sci. Libr. 349, 457-472 (2008), arXiv:gr-qc/0509114 [gr-qc].

[117] E. V. Pitjeva and N. P. Pitjev, "Relativistic effects and dark matter in the Solar system from observations of planets and spacecraft," Mon. Not. Roy. Astron. Soc. 432, 3431 (2013), arXiv:1306.3043 [astro-ph.EP].

[118] Radouane Gannouji, David Polarski, Andre Ranquet, and Alexei A. Starobinsky, "Scalar-Tensor Models of Normal and Phantom Dark Energy," JCAP 0609, 016 (2006), arXiv:astro-ph/0606287 [astro-ph].

[119] S. Nesseris and Leandros Perivolaropoulos, "The Limits of Extended Quintessence," Phys. Rev. D75, 023517 (2007), arXiv:astro-ph/0611238 [astro-ph].

[120] P. A. R. Ade et al. (Planck), "Planck 2015 results. XIV. Dark energy and modified gravity," Astron. Astrophys. 594, A14 (2016), arXiv:1502.01590 [astro-ph.CO].

[121] Eleonora Di Valentino, Alessandro Melchiorri, and Joseph Silk, "Cosmological hints of modified gravity?" Phys. Rev. D93, 023513 (2016), arXiv:1509.07501 [astro-ph.CO].

[122] Radouane Gannouji, Lavrentios Kazantzidis, Leandros Perivolaropoulos, and David Polarski, "Consistency of modified gravity with a decreasing $G_{\text {eff }}(z)$ in a

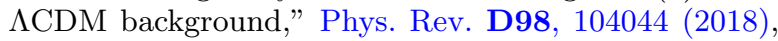
arXiv:1809.07034 [gr-qc].

[123] Eric V. Linder, "No Slip Gravity," JCAP 1803, 005 (2018), arXiv:1801.01503 [astro-ph.CO].

[124] Rubén Arjona, Wilmar Cardona, and Savvas Nesseris, 
"Designing Horndeski and the effective fluid approach," Phys. Rev. D100, 063526 (2019), arXiv:1904.06294 [astro-ph.CO].

[125] Shinji Tsujikawa, "Possibility of realizing weak gravity in redshift space distortion measurements," Phys. Rev. D92, 044029 (2015), arXiv:1505.02459 [astro-ph.CO].

[126] Luca Amendola, Dario Bettoni, Ana Marta Pinho, and Santiago Casas, "Measuring gravity at cosmological scales," Universe 6, 20 (2020), arXiv:1902.06978 [astroph.CO].

[127] Azadeh Moradinezhad Dizgah and Ruth Durrer, "Lensing corrections to the $E_{g}(z)$ statistics from large scale structure," JCAP 1609, 035 (2016), arXiv:1604.08914 [astro-ph.CO].

[128] Basundhara Ghosh and Ruth Durrer, "The observable $E_{g}$ statistics," JCAP 1906, 010 (2019), arXiv:1812.09546 [astro-ph.CO].

[129] M. A. Troxel et al. (DES), "Dark Energy Survey Year 1 results: Cosmological constraints from cosmic shear," Phys. Rev. D98, 043528 (2018), arXiv:1708.01538 [astro-ph.CO].

[130] A. Drlica-Wagner et al. (DES), "Dark Energy Survey Year 1 Results: Photometric Data Set for Cosmology," Astrophys. J. Suppl. 235, 33 (2018), arXiv:1708.01531 [astro-ph.CO].

[131] B. Hoyle et al. (DES), "Dark Energy Survey Year 1 Results: Redshift distributions of the weak lensing source galaxies," Mon. Not. Roy. Astron. Soc. 478, 592-610 (2018), arXiv:1708.01532 [astro-ph.CO].

[132] J. Elvin-Poole et al. (DES), "Dark Energy Survey year 1 results: Galaxy clustering for combined probes," Phys. Rev. D98, 042006 (2018), arXiv:1708.01536 [astroph.CO].

[133] Luca Amendola et al., "Cosmology and fundamental physics with the Euclid satellite," Living Rev. Rel. 21, 2 (2018), arXiv:1606.00180 [astro-ph.CO].

[134] http://leandros.physics.uoi.gr/egfs8plots.zip.

[135] Yong-Seon Song and Will J. Percival, "Reconstructing the history of structure formation using Redshift Distortions," JCAP 0910, 004 (2009), arXiv:0807.0810 [astro$\mathrm{ph}]$.

[136] Max Tegmark et al. (SDSS), "Cosmological Constraints from the SDSS Luminous Red Galaxies," Phys. Rev. D74, 123507 (2006), arXiv:astro-ph/0608632 [astro-ph].

[137] Marc Davis, Adi Nusser, Karen Masters, Christopher Springob, John P. Huchra, and Gerard Lemson, "Local Gravity versus Local Velocity: Solutions for $\beta$ and nonlinear bias," Mon. Not. Roy. Astron. Soc. 413, 2906 (2011), arXiv:1011.3114 [astro-ph.CO].

[138] Michael J. Hudson and Stephen J. Turnbull, "The growth rate of cosmic structure from peculiar velocities at low and high redshifts," The Astrophysical Journal Letters 751, L30 (2012).

[139] Stephen J. Turnbull, Michael J. Hudson, Hume A. Feldman, Malcolm Hicken, Robert P. Kirshner, and Richard Watkins, "Cosmic flows in the nearby universe from type ia supernovae," Monthly Notices of the Royal Astronomical Society 420, 447-454 (2012).

[140] L. Samushia, W. J. Percival, and A. Raccanelli, "Interpreting large-scale redshift-space distortion measurements," Monthly Notices of the Royal Astronomical Society 420, 2102-2119 (2012).

[141] C. Blake, S. Brough, M. Colless, C. Contreras, W. Couch, S. Croom, D. Croton, T. M. Davis,
M. J. Drinkwater, K. Forster, D. Gilbank, M. Gladders, K. Glazebrook, B. Jelliffe, R. J. Jurek, I.-h. Li, B. Madore, D. C. Martin, K. Pimbblet, G. B. Poole, M. Pracy, R. Sharp, E. Wisnioski, D. Woods, T. K. Wyder, and H. K. C. Yee, "The WiggleZ Dark Energy Survey: joint measurements of the expansion and growth history at $z 1, "$ mnras 425, 405-414 (2012), arXiv:1204.3674.

[142] Rita Tojeiro, Will J. Percival, Jon Brinkmann, Joel R. Brownstein, Daniel J. Eisenstein, Marc Manera, Claudia Maraston, Cameron K. McBride, Demitri Muna, Beth Reid, Ashley J. Ross, Nicholas P. Ross, Lado Samushia, Nikhil Padmanabhan, Donald P. Schneider, Ramin Skibba, Ariel G. Sánchez, Molly E. C. Swanson, Daniel Thomas, Jeremy L. Tinker, Licia Verde, David A. Wake, Benjamin A. Weaver, and Gong-Bo Zhao, "The clustering of galaxies in the sdss-iii baryon oscillation spectroscopic survey: measuring structure growth using passive galaxies," Monthly Notices of the Royal Astronomical Society 424, 2339-2344 (2012).

[143] S. de la Torre et al., "The VIMOS Public Extragalactic Redshift Survey (VIPERS). Galaxy clustering and redshift-space distortions at $\mathrm{z}=0.8$ in the first data release," Astron. Astrophys. 557, A54 (2013), arXiv:1303.2622 [astro-ph.CO].

[144] Chia-Hsun Chuang and Yun Wang, "Modelling the anisotropic two-point galaxy correlation function on small scales and single-probe measurements of $h(z)$, $d a(z)$ and $f(z) \sigma 8(z)$ from the sloan digital sky survey dr7 luminous red galaxies," Monthly Notices of the Royal Astronomical Society 435, 255-262 (2013).

[145] E. Komatsu et al. (WMAP), "Seven-Year Wilkinson Microwave Anisotropy Probe (WMAP) Observations: Cosmological Interpretation," Astrophys. J. Suppl. 192, 18 (2011), arXiv:1001.4538 [astro-ph.CO].

[146] Chris Blake et al., "Galaxy And Mass Assembly (GAMA): improved cosmic growth measurements using multiple tracers of large-scale structure," Mon. Not. Roy. Astron. Soc. 436, 3089 (2013), arXiv:1309.5556 [astro-ph.CO].

[147] Ariel G. Sanchez et al., "The clustering of galaxies in the SDSS-III Baryon Oscillation Spectroscopic Survey: cosmological implications of the full shape of the clustering wedges in the data release 10 and 11 galaxy samples," Mon. Not. Roy. Astron. Soc. 440, 2692-2713 (2014), arXiv:1312.4854 [astro-ph.CO].

[148] Lauren Anderson et al. (BOSS), "The clustering of galaxies in the SDSS-III Baryon Oscillation Spectroscopic Survey: baryon acoustic oscillations in the Data Releases 10 and 11 Galaxy samples," Mon. Not. Roy. Astron. Soc. 441, 24-62 (2014), arXiv:1312.4877 [astroph.CO].

[149] Cullan Howlett, Ashley Ross, Lado Samushia, Will Percival, and Marc Manera, "The clustering of the SDSS main galaxy sample - II. Mock galaxy catalogues and a measurement of the growth of structure from redshift space distortions at $z=0.15$," Mon. Not. Roy. Astron. Soc. 449, 848-866 (2015), arXiv:1409.3238 [astroph.CO].

[150] Martin Feix, Adi Nusser, and Enzo Branchini, "Growth Rate of Cosmological Perturbations at $z \approx 0.1$ from a New Observational Test," Phys. Rev. Lett. 115, 011301 (2015), arXiv:1503.05945 [astro-ph.CO].

[151] Max Tegmark et al. (SDSS), "The 3-D power spectrum 
of galaxies from the SDSS," Astrophys. J. 606, 702-740 (2004), arXiv:astro-ph/0310725 [astro-ph].

[152] Teppei Okumura et al., "The Subaru FMOS galaxy redshift survey (FastSound). IV. New constraint on gravity theory from redshift space distortions at $z \sim 1.4$," Publ. Astron. Soc. Jap. 68, 24 (2016), arXiv:1511.08083 [astro-ph.CO].

[153] G. Hinshaw et al. (WMAP), "Nine-Year Wilkinson Microwave Anisotropy Probe (WMAP) Observations: Cosmological Parameter Results," Astrophys. J. Suppl. 208, 19 (2013), arXiv:1212.5226 [astro-ph.CO].

[154] Chia-Hsun Chuang et al., "The clustering of galaxies in the SDSS-III Baryon Oscillation Spectroscopic Survey: single-probe measurements from CMASS anisotropic galaxy clustering," Mon. Not. Roy. Astron. Soc. 461, 3781-3793 (2016), arXiv:1312.4889 [astro-ph.CO].

[155] Shadab Alam et al. (BOSS), "The clustering of galaxies in the completed SDSS-III Baryon Oscillation Spectroscopic Survey: cosmological analysis of the DR12 galaxy sample," Mon. Not. Roy. Astron. Soc. 470, 2617-2652 (2017), arXiv:1607.03155 [astro-ph.CO].

[156] Florian Beutler et al. (BOSS), "The clustering of galaxies in the completed SDSS-III Baryon Oscillation Spectroscopic Survey: Anisotropic galaxy clustering in Fourier-space," Mon. Not. Roy. Astron. Soc. 466, 22422260 (2017), arXiv:1607.03150 [astro-ph.CO].

[157] Michael J. Wilson, Geometric and growth rate tests of General Relativity with recovered linear cosmological perturbations, Ph.D. thesis, Edinburgh U. (2016), arXiv:1610.08362 [astro-ph.CO].

[158] Héctor Gil-Marín, Will J. Percival, Licia Verde, Joel R. Brownstein, Chia-Hsun Chuang, Francisco-Shu Kitaura, Sergio A. Rodríguez-Torres, and Matthew D. Olmstead, "The clustering of galaxies in the SDSS-III Baryon Oscillation Spectroscopic Survey: RSD measurement from the power spectrum and bispectrum of the DR12 BOSS galaxies," Mon. Not. Roy. Astron. Soc. 465, 1757-1788 (2017), arXiv:1606.00439 [astroph.CO].

[159] A. J. Hawken et al., "The VIMOS Public Extragalactic Redshift Survey: Measuring the growth rate of structure around cosmic voids," Astron. Astrophys. 607, A54 (2017), arXiv:1611.07046 [astro-ph.CO].

[160] Dragan Huterer, Daniel Shafer, Daniel Scolnic, and Fabian Schmidt, "Testing $\Lambda$ CDM at the lowest redshifts with SN Ia and galaxy velocities," JCAP 1705, 015 (2017), arXiv:1611.09862 [astro-ph.CO].

[161] A. Pezzotta et al., "The VIMOS Public Extragalactic Redshift Survey (VIPERS): The growth of structure at $0.5<z<1.2$ from redshift-space distortions in the clustering of the PDR-2 final sample," Astron. Astrophys. 604, A33 (2017), arXiv:1612.05645 [astro-ph.CO].

[162] Martin Feix, Enzo Branchini, and Adi Nusser, "Speed from light: growth rate and bulk flow at $z \approx 0.1$ from improved SDSS DR13 photometry," Mon. Not. Roy. Astron. Soc. 468, 1420-1425 (2017), arXiv:1612.07809 [astro-ph.CO].

[163] Cullan Howlett, Lister Staveley-Smith, Pascal J. Elahi, Tao Hong, Tom H. Jarrett, D. Heath Jones, Bärbel S. Koribalski, Lucas M. Macri, Karen L. Masters, and Christopher M. Springob, "2MTF VI. Measuring the velocity power spectrum," Mon. Not. Roy. Astron. Soc. 471, 3135 (2017), arXiv:1706.05130 [astro-ph.CO].

[164] F. G. Mohammad et al., "The VIMOS Public Extra- galactic Redshift Survey (VIPERS): An unbiased estimate of the growth rate of structure at $\langle\mathbf{z}\rangle=\mathbf{0 . 8 5}$ using the clustering of luminous blue galaxies," (2017), arXiv:1708.00026 [astro-ph.CO].

[165] Yuting Wang, Gong-Bo Zhao, Chia-Hsun Chuang, Marcos Pellejero-Ibanez, Cheng Zhao, Francisco-Shu Kitaura, and Sergio Rodriguez-Torres, "The clustering of galaxies in the completed SDSS-III Baryon Oscillation Spectroscopic Survey: a tomographic analysis of structure growth and expansion rate from anisotropic galaxy clustering," (2017), arXiv:1709.05173 [astro-ph.CO].

[166] Feng Shi et al., "Mapping the Real Space Distributions of Galaxies in SDSS DR7: II. Measuring the growth rate, linear mass variance and biases of galaxies at redshift 0.1," (2017), arXiv:1712.04163 [astro-ph.CO].

[167] Héctor Gil-Marín et al., "The clustering of the SDSSIV extended Baryon Oscillation Spectroscopic Survey DR14 quasar sample: structure growth rate measurement from the anisotropic quasar power spectrum in the redshift range $0.8<z<2.2$," Mon. Not. Roy. Astron. Soc. 477, 1604-1638 (2018), arXiv:1801.02689 [astroph.CO].

[168] Jiamin Hou et al., "The clustering of the SDSSIV extended Baryon Oscillation Spectroscopic Survey DR14 quasar sample: anisotropic clustering analysis in configuration-space," (2018), arXiv:1801.02656 [astroph.CO].

[169] Gong-Bo Zhao et al., "The clustering of the SDSS-IV extended Baryon Oscillation Spectroscopic Survey DR14 quasar sample: a tomographic measurement of cosmic structure growth and expansion rate based on optimal redshift weights," (2018), arXiv:1801.03043 [astroph.CO].

[170] F. G. Mohammad et al., "The VIMOS Public Extragalactic Redshift Survey (VIPERS): Unbiased clustering estimate with VIPERS slit assignment," Astron. Astrophys. 619, A17 (2018), arXiv:1807.05999 [astroph.CO].

[171] Seshadri Nadathur, Paul M. Carter, Will J. Percival, Hans A. Winther, and Julian Bautista, "Beyond BAO: Improving cosmological constraints from BOSS data with measurement of the void-galaxy cross-correlation," Phys. Rev. D100, 023504 (2019), arXiv:1904.01030 [astro-ph.CO].

[172] Fei Qin, Cullan Howlett, and Lister Staveley-Smith, "The redshift-space momentum power spectrum - II. Measuring the growth rate from the combined $2 \mathrm{MTF}$ and 6dFGSv surveys," Mon. Not. Roy. Astron. Soc. 487, 5235-5247 (2019), arXiv:1906.02874 [astro-ph.CO].

[173] M. Icaza-Lizaola et al., "The clustering of the SDSSIV extended Baryon Oscillation Spectroscopic Survey DR14 LRG sample: structure growth rate measurement from the anisotropic LRG correlation function in the redshift range 0.6 ; z i 1.0," (2019), arXiv:1909.07742 [astro-ph.CO].

[174] A. Amon et al., "KiDS+2dFLenS+GAMA: Testing the cosmological model with the $E_{\mathrm{G}}$ statistic," Mon. Not. Roy. Astron. Soc. 479, 3422-3437 (2018), arXiv:1711.10999 [astro-ph.CO].

[175] Sukhdeep Singh, Shadab Alam, Rachel Mandelbaum, Uros Seljak, Sergio Rodriguez-Torres, and Shirley Ho, "Probing gravity with a joint analysis of galaxy and CMB lensing and SDSS spectroscopy," Mon. Not. Roy. Astron. Soc. 482, 785-806 (2019), arXiv:1803.08915 
[astro-ph.CO].

[176] Chris Blake et al., "RCSLenS: Testing gravitational physics through the cross-correlation of weak lensing and large-scale structure," Mon. Not. Roy. Astron. Soc. 456, 2806-2828 (2016), arXiv:1507.03086 [astroph.CO].

[177] Shadab Alam, Hironao Miyatake, Surhud More, Shirley Ho, and Rachel Mandelbaum, "Testing gravity on large scales by combining weak lensing with galaxy clustering using CFHTLenS and BOSS CMASS," Mon. Not. Roy. Astron. Soc. 465, 4853-4865 (2017), arXiv:1610.09410 [astro-ph.CO].

[178] E. Jullo et al., "Testing gravity with galaxy-galaxy lensing and redshift-space distortions using CFHT-Stripe 82, CFHTLenS and BOSS CMASS datasets," Astron. Astrophys. 627, A137 (2019), arXiv:1903.07160 [astroph.CO]. 